\title{
Synthesis, Evaluation and Docking Study of 1, 3, 5-Triazine Derivatives as Cytotoxic Agents against Lung Cancer
}

\author{
Marwa F. Balaha ${ }^{a}$, Mervat H. El-Hamamsy ${ }^{b^{*}}$, Nabawya A. Sharaf El-Din ${ }^{c}$, Nageh A.El-Mahdy ${ }^{d}$ \\ ${ }^{a}$ Department of Pharmaceutical Chemistry, Faculty of Pharmacy, Kafrelsheikh University, Kafrelsheikh, Egypt. ${ }^{\mathrm{b}}$ Department of Pharmaceutical Chemistry, \\ Faculty of Pharmacy, Tanta University, El Giesh street, 31527, Tanta, Egypt. ${ }^{c}$ Department of Pharmaceutical Chemistry, Faculty of Pharmacy, Tanta \\ University, El Giesh street, 31527, Tanta, Egypt. ${ }^{\mathrm{d} D e p a r t m e n t}$ of Pharmacology and toxicology, Faculty of Pharmacy, Tanta University, El Giesh street, \\ 31527, Tanta, Egypt.
}

\section{ARTICLE INFO}

Article history:

Received on: 01/12/2015

Revised on: 09/01/2016

Accepted on: 12/02/2016

Available online: $30 / 04 / 2016$

\section{Key words:}

1,3,5-triazine; synthesis; anticancer agent;

dihydrofolate reductase;

molecular docking.

\begin{abstract}
Knowing that dihydrofolate reductase (DHFR) is the primary target enzyme for antifolate drugs and 1,3,5triazine derivatives containing various amino groups at position 2, 4 or 6 have been known as potent anticancer drugs, two series of tri-amino-substituted 1,3,5-triazine derivatives were designed, synthesized and evaluated as cytotoxic agents against non-small cell lung cancer (A549). The first series are $\mathrm{N}^{2}$-(4-phenylthiazol-2-yl)-1,3,5triazine-2,4,6-triamine analogs and the second series are4-((4,6-Diamino-1,3,5-triazin-2-yl)amino)-4H-1,2,4triazole-3-thiol analogs. Out of twenty two synthesized compounds there were thirteen compounds showed a higher cytotoxic activity against A549 cell line than methotrexate and four compounds were equipotent to methotrexate. Compounds $8 \mathrm{e}, 9 \mathrm{a}, 10 \mathrm{e}$ and $11 \mathrm{e}$ showed the highest cytotoxic activity with $\mathrm{IC}_{50}$ values of 50,42 , 62 and $28 \mathrm{nM}$ respectively. Molecular docking study was performed to interpret the comparative differences in the binding interactions of the synthesized novel compounds at molecular level as inhibitors of human dihydrofolate reductase (hDHFR)and to understand the structure activity relationships. The excellent anticancer activity of synthesized analogs presented in this study needs further investigation as highly promising cytotoxic lead agents against lung cancer.
\end{abstract}

\section{INTRODUCTION}

Cancer is one of the leading causes of death in the world, particularly in developing countries (WHO Fact sheet on cancer, 2015). It accounts for 8.2 million deaths in 2012 (WHO, 2012, 2015). Worldwide, lung cancer is the most common cancer among men in terms of both incidence and mortality, while among women has the third highest incidence, and is second after breast cancer in mortality (WHO, World Cancer Report 2014, 2015). Dihydrofolate reductase (DHFR) inhibition has long been identified as an important target for the development of chemotherapeutic agents against bacterial and parasitic infections as well as cancer(Anderson and Wright, 2014; Al-Rashood et al., 2014).This considerable pharmacological interest in DHFR is due to that enzyme inhibition results in depletion of intracellular reduced folates necessary for one-carbon transfer reactions which, in turn, are important for the biosynthesis of thymidylate,

\footnotetext{
* Corresponding Author

E-mail: mhamamsy@pharm.tanta.edu.eg
}

purine nucleotides, methionine and many other compounds necessary for RNA, DNA, and protein synthesis (Al-Rashood et al., 2014). In addition, this enzyme has high binding affinities and selectivity towards the substrate analogs that are not readily displaced by the natural substrates.

This made the DHFR as an ideal target for rational and efficient drug design (Sunduru et al., 2010). Triazines have a wide range of biological activities (Patel et al., 2012) including, antimicrobial (Ma et al., 2011), antifungal (Sarmah et al., 2012), antimalarial activity (Manohar et al., 2013), antiviral activity (Mibu et al., 2014) and cytotoxic activity (El-Hamamsy and ElMahdy, 2014; Zhu et al., 2012).

Several studies based on the triazine scaffold toward antitumor activity have been carried out (Ma and Chui, 2010) starting by Baker who studied active site-directed inhibition of hDHFR enzyme (Baker and Lourens, 1967). These compounds are inhibitors of DHFR and are clinically promising for use in cancer chemotherapy (Camerman et al., 1978).

Hexamethylmelamine (Altretamine) is 1,3,5-triazine derivative and is used clinically as antitumor agent against lung, 
ovarian and breast cancers(Kumar et al., 2014).1,3,5-triazine derivatives containing various amino groups at position 2,4 or 6 have been known as anticancer drugs (Sączewski et al., 2006). Aiming at discovering new antitumor agents, two different novel series of 1,3,5-triazine derivatives were designed, synthesized and evaluated as potential cytotoxic agents against non-small cell lung cancer (A549). The first series was designed to have a substituted thiazole ring (series $\mathrm{A}$ ) while the second series would contain a substituted triazole ring (series B) as shown in Figure 1. Two model compounds with phenyl ring would also be synthesized to be compared with the activity of our target compounds (Figure 1).Different strategies have been employed for the synthesis of mono, di- or tri-substituted triazines (Daniel et al., 2004). The most important starting compound for preparing substituted triazines was the commercially available, cyanuric chloride which was very inexpensive (Blotny, 2006). The reactivity of its chlorine atoms towards nucleophiles was controlled by temperature.

Molecular docking study was performed for synthesized compounds along with the reference molecule, methotrexate (MTX) into the active site of hDHFR enzyme using FlexX module in LeadIT 2.1.8 software-package (BioSolveIT, 2014). Molecular docking study aims to interpret the comparative differences in the binding interactions of the synthesized novel compounds at molecular level as inhibitors of hDHFR to understand the structure activity relationships.

\section{MATERIAL AND METHODS}

\section{Chemistry}

Melting points were determined on a Stuart SMP10 capillary melting point apparatus and are uncorrected. Elemental analyses were performed by Main Defense Chemical Laboratory, 17 Km Cairo-Suez Road, Almaza, Cairo, Egypt. New compounds were analyzed for $\mathrm{C}, \mathrm{H}, \mathrm{N}, \mathrm{S}$ and were within $\pm 0.4 \%$ of the theoretical values. Nuclear magnetic resonance (NMR) spectra were recorded on a BRUKER (400 MHz) spectrometer, faculty of science, Kafrelsheikh University, Kafrelsheikh, Egypt. Chemical shifts are expressed in $\delta \mathrm{ppm}$ with reference to tetramethylsilane (TMS). All organic reagents used were obtained from SigmaAldrich Chemical Company, Germany and were used without further purification. Silica gel chromatographic sheets were used for thin layer chromatography (TLC) using pre coated aluminumbacked plates (Merck, silica gel $60 \quad F_{254}$ ). Compounds were visualized with $254 \mathrm{~nm}$ UV lamp and the eluent used was chloroform: methanol (9:1).

\section{General procedures for synthesis of compounds 1a-c}

Suspend $3.8 \mathrm{~g}(50 \mathrm{mmol})$ thiourea in $10 \mathrm{ml}$ water. Add $(50 \mathrm{mmol})$ of 4-substituted phenacyl bromide over a period of 30 minutes. Thiourea dissolved as the reaction proceeds and the yellow solution was heated under reflux for $2 \mathrm{~h}$. After cooling the reaction mixture in an ice bath add with stirring $2 \mathrm{~g}$ of solid sodium hydroxide. The formed solid was collected, washed several times with distilled water to remove excess of alkali and recrystallized from aqueous ethanol (El-Din et al,. 1998).

\section{General procedures for synthesis of compounds 1d-e}

Thiourea $7.6 \mathrm{~g}$ (100 mmole) and iodine $(12.7 \mathrm{~g}$ (50mmole) were triturated and mixed with 4-substituted acetophenone $(50 \mathrm{mmole})$ in dimethyl formamide $(50 \mathrm{ml})$. The mixture was heated on water bath with stirring for $30 \mathrm{~h}$. The heated solution was poured in distilled water; and the formed precipitate was filtered off and recrystallized from aqueous ethanol (King and Hlavacek, 1950).

\section{Thiocarbohydrazide (2)}

To a vigorously stirred solution of $(125 \mathrm{~g}, 2.5 \mathrm{~mol})$ hydrazine hydrate in $75 \mathrm{ml}$ of water, carbon disulfide (38 g, 0.5 mole) was added drop wise. The reaction mixture was heated at reflux for $30 \mathrm{~min}$, cooled in an ice bath for $30 \mathrm{~min}$ and the precipitated colorless crystals was filtered off, washed with ethanol and ether, and dried. The mother liquor was returned to the reaction flask and the process repeated twice, after which no more thiocarbohydrazide was obtained. The obtained product recrystalized from the minimum amount of water acidified with few drops of conc. HClas colorless crystals with yield of $(45.10 \mathrm{~g}$, $85 \%)$, melting point $\left(170-171^{\circ} \mathrm{C}\right)$ which is agreed with the reported data (Audrieth et al., 1954).

\section{General procedures for synthesis of compounds $3 a-d$}

Thiocarbohydrazide (2) $(5.3 \mathrm{~g}, 50 \mathrm{mmol})$ was stirred with the appropriate carboxylic acid $(10 \mathrm{ml})$ and was heated to boiling for $20 \mathrm{~min}$. After cooling the reaction mixture was diluted with ethyl acetate to give a white precipitate. The precipitate was filtered, washed with water and dried. The products were crystalized from aqueous ethanol (El-Din et al,. 1998).

\section{Benzhydrazide (4)}

Benzhydrazide was synthesized by heating ethyl benzoate $(30.0 \mathrm{~g}, 0.2 \mathrm{~mol})$ with $98 \%$ hydrazine hydrate $(50 \mathrm{~g}, 1$ mol) under reflux for $3 \mathrm{~h}$. The reaction mixture was cooled and diluted with water. The separated solid was filtered and crystallized from ethanol. Benzhydrazide (5) was obtained as light brown crystals with a yield of $(8.44 \mathrm{~g}, 31 \%)$, melting point (111$113{ }^{\circ} \mathrm{C}$ ) which agreed with the reported data (Heilbron and Bunbury, 1934).

\section{Potassium 3-benzoyldithiocarbazate (5)}

To an ice cooled solution of potassium hydroxide (8.41 g, $0.15 \mathrm{~mol})$ in absolute ethanol $(200 \mathrm{ml})$ containing the benzhydrazide (5) (13.61 g, $0.1 \mathrm{~mol})$, carbon disulfide(11.4 g, 0.15 mole) was added drop wise. The mixture was diluted with absolute ethanol $(150 \mathrm{ml})$, and stirred at room temperature for $16 \mathrm{~h}$. Dry ether $(200 \mathrm{ml})$ was then added and the separated solid was filtered, washed with ether and dried. The product obtained in nearly quantitative yield and was employed in next reactions without further purification (Reid and Heindel, 1976; El-Din et al,. 1998).

\section{5-phenyl-4-amino-s-triazole-3-thiol (3e)}

A suspension of potassium-3-benzoyldithiocarbazate (4) $(5.0 \mathrm{~g}, 0.02 \mathrm{~mol}), 98 \%$ hydrazine hydrate $(2 \mathrm{~g}, 0.04 \mathrm{~mol})$ in water 
$(20 \mathrm{ml})$ were heated under reflux while stirring for $1 \mathrm{~h}$. Cold water $(100 \mathrm{ml})$ was added and the mixture neutralized with concentrated hydrochloric acid. The separated solid product was filtered, washed with cold water, dried and crystalized from aqueous ethanol. Compound (3e) (2. $89 \mathrm{~g}, 75 \%)$ was obtained as white solid, melting point $\left(204-206^{\circ} \mathrm{C}\right)$ which is agreed with the reported data (Reid and Heindel, 1976; El-Din et al,. 1998).

\section{General procedures for synthesis of compounds 6a-6e}

To a stirring suspension of $(10 \mathrm{mmol})$ cyanuric chloride and $(10 \mathrm{mmol})$ potassium carbonate in methylene chloride. The corresponding 4-(4-substitutedphenyl)thiazol-2-amine (1a-1e) (10 mmol) was added. The reaction mixture was left $72 \mathrm{~h}$ at room temperature. The reaction mixture was partitioned between methylene chloride $(250 \mathrm{ml})$ and $\mathrm{HCl}(0.1 \mathrm{M}, 500 \mathrm{ml})$. The collected organic layer was washed with dist. water $(3 \times 75 \mathrm{ml})$, brine solution $(50 \mathrm{ml})$, dried over anhydrous magnesium sulfate and then evaporated under vacuum to obtain the pure product.

\section{N-(4,6-dichloro-1,3,5-triazin-2-yl)-4-phenylthiazol-2-amine (6a)}

From cyanuric chloride $(1.84 \mathrm{~g}, 10 \mathrm{mmol})$, potassium carbonate (1.38 g, $10 \mathrm{mmol}$ ) and compound (1a) (1.76 g, $10 \mathrm{mmol})$, compound (6a) $(2.1 \mathrm{~g}, 64.8 \%)$ was obtained as light green solid, melting point $\left(159-161^{\circ} \mathrm{C}\right),{ }^{1} \mathrm{H}-\mathrm{NMR}$ (DMSO-d $\left.\mathrm{d}_{6}, \mathrm{ppm}\right)$ $\delta$ : $6.77(1 \mathrm{H}, \mathrm{s}, \mathrm{CH}$ of thiazole ring), $7.36(1 \mathrm{H}, \mathrm{m}, \mathrm{Ph} 4-\mathrm{H}), 7.44$ $\left(2 \mathrm{H}, \mathrm{t}, J=7.6 \mathrm{~Hz}, \mathrm{Ph} 3,5-\mathrm{H}_{2}\right), 7.66\left(2 \mathrm{H}, \mathrm{d}, J=7.6 \mathrm{~Hz}, \mathrm{Ph} 2,6-\mathrm{H}_{2}\right)$, $8.37(1 \mathrm{H}, \mathrm{s}, \mathrm{NH})$.

\section{4-(4-Chlorophenyl)-N-(4,6-dichloro-1,3,5-triazin-2-yl)thiazol-2- amine (6b)}

From cyanuric chloride $(1.84 \mathrm{~g}, 10 \mathrm{mmol})$, potassium carbonate $(1.38 \mathrm{~g}, 10 \mathrm{mmol})$ and compound (1b) $(2.1 \mathrm{~g}, 10 \mathrm{mmol})$, compound (6b) $(2.4 \mathrm{~g}, 68 \%)$ was obtained as light yellow solid, melting point $\left(169-171^{\circ} \mathrm{C}\right),{ }^{1} \mathrm{H}-\mathrm{NMR}$ (DMSO-d $\left.6, \mathrm{ppm}\right) \delta: 7.28$ $(1 \mathrm{H}, \mathrm{s}, \mathrm{CH}$ of thiazole ring), $7.43(1 \mathrm{H}, \mathrm{s}, \mathrm{NH}), 7.47(2 \mathrm{H}, \mathrm{d}, J=7.6$ $\left.\mathrm{Hz}, \mathrm{Ph} 3,5-\mathrm{H}_{2}\right), 7.63\left(2 \mathrm{H}, \mathrm{d}, J=7.6 \mathrm{~Hz}, \mathrm{Ph} 2,6-\mathrm{H}_{2}\right)$.

\section{$N$-(4,6-dichloro-1,3,5-triazin-2-yl)-4-(4-methoxyphenyl)thiazol-2- amine (6c)}

From cyanuric chloride $(1.84 \mathrm{~g}, 10 \mathrm{mmol})$, potassium carbonate $(1.38 \mathrm{~g}, 10 \mathrm{mmol})$ and compound (1c) (2.06 g, 10 mmol), compound (6c) (2.3 g, $65 \%$ ) was obtained as yellow solid, melting point $\left(184-186^{\circ} \mathrm{C}\right),{ }^{1} \mathrm{H}-\mathrm{NMR}$ (DMSO-d 6 , ppm) $\delta: 3.77(3 \mathrm{H}$, $\left.\mathrm{s}, \mathrm{OCH}_{3}\right), 6.98(1 \mathrm{H}, \mathrm{s}, \mathrm{CH}$ of thiazole ring $), 7.05(2 \mathrm{H}, \mathrm{d}, J=7.6 \mathrm{~Hz}$, $\left.\mathrm{Ph} 3,5-\mathrm{H}_{2}\right), 7.61\left(2 \mathrm{H}, \mathrm{d}, J=7.6 \mathrm{~Hz}, \mathrm{Ph} 2,4-\mathrm{H}_{2}\right), 8.53(1 \mathrm{H}, \mathrm{s}, \mathrm{NH})$. $N$-(4,6-dichloro-1,3,5-triazin-2-yl)-4-(p-tolyl)thiazol-2-amine (6d) From cyanuric chloride $(1.84 \mathrm{~g}, 10 \mathrm{mmol})$, potassium carbonate (1.38 g, $10 \mathrm{mmol})$ and compound (1d) (1.90 g, $10 \mathrm{mmol})$, compound (6d) $(2.36 \mathrm{~g}, 70 \%)$ was obtained as light green solid, melting point $\left(155-156^{\circ} \mathrm{C}\right),{ }^{1} \mathrm{H}-\mathrm{NMR}$ (DMSO-d $\left.\mathrm{d}_{6}, \mathrm{ppm}\right) \delta: 2.32$ $\left(3 \mathrm{H}, \mathrm{s}, \mathrm{CH}_{3}\right), 7.13(1 \mathrm{H}, \mathrm{s}, \mathrm{CH}$ of thiazole ring), $7.24(1 \mathrm{H}, \mathrm{s}, \mathrm{NH})$, $7.31\left(2 \mathrm{H}, \mathrm{Ph} \mathrm{d}, J=7.6 \mathrm{~Hz}, 3,5-\mathrm{H}_{2}\right), 7.61(2 \mathrm{H}, \mathrm{d}, J=7.6 \mathrm{~Hz}, \mathrm{Ph} 2,4-$ $\mathrm{H}_{2}$ ).
$N$-(4,6-dichloro- 1,3,5-triazin-2-yl)-4-(4-nitrophenyl)thiazol-2amine (6e)

From cyanuric chloride $(1.84 \mathrm{~g}, 10 \mathrm{mmol})$, potassium carbonate $(1.38 \mathrm{~g}, 10 \mathrm{mmol})$ and compound (1e) $(2.21 \mathrm{~g}, 10$ mmol), compound (6e) $(2.28 \mathrm{~g}, 61.9 \%)$ was obtained as brown solid, melting point $\left(208-210{ }^{\circ} \mathrm{C}\right),{ }^{1} \mathrm{H}-\mathrm{NMR}$ (DMSO-d $\left.\mathrm{D}_{6}, \mathrm{ppm}\right) \delta$ : $7.25(1 \mathrm{H}, \mathrm{s}, \mathrm{CH}$ of thiazole ring), $7.93(2 \mathrm{H}, \mathrm{d}, J=7.8 \mathrm{~Hz}, \mathrm{Ph} 2,6-$ $\left.\mathrm{H}_{2}\right), 8.37\left(2 \mathrm{H}, \mathrm{d}, J=7.8 \mathrm{~Hz}, \mathrm{Ph} 3,5-\mathrm{H}_{2}\right), 8.96(1 \mathrm{H}, \mathrm{s}, \mathrm{NH})$.

\section{General procedures for synthesis of compounds 7a-7e}

To a stirring suspension of (20 mmol) cyanuric chloride and $(20 \mathrm{mmol})$ potassium carbonate in methylene chloride, (10mmol) of the corresponding 4-amino-5-substituted-4H-1,2,4triazole-3-thiol (2a-e) was added. The reaction mixture was stirred for (24-48) $\mathrm{h}$ at room temperature. The reaction mixture was partitioned between methylene chloride $(250 \mathrm{ml})$ and $\mathrm{HCl}(0.1 \mathrm{M}$, $500 \mathrm{ml})$. The collected organic layer was washed with dist. water $(3 \times 75 \mathrm{ml})$, brine solution $(50 \mathrm{ml})$, dried over anhydrous magnesium sulfate and then evaporated under vacuum to obtain the pure product.

\section{4-((4,6-Dichloro-1,3,5-triazin-2-yl )amino)-4H-1,2,4-triazole-3- thiol (7a)}

From cyanuric chloride $(3.68 \mathrm{~g}, 20 \mathrm{mmol})$, potassium carbonate (2.76 g, $20 \mathrm{mmol}$ ) and compound (2a) (2.32 g, 20 mmol), compound (7a) was obtained as off white solid with yield of $(2.28 \mathrm{~g}, 31 \%)$ after $48 \mathrm{~h}$, melting point $\left(180-181^{\circ} \mathrm{C}\right),{ }^{1} \mathrm{H}-\mathrm{NMR}$ $\left(\right.$ DMSO-d $\left._{6}, \mathrm{ppm}\right) \delta: 8.09(1 \mathrm{H}, \mathrm{s}, \mathrm{CH}$ of triazole ring $), 8.96(1 \mathrm{H}, \mathrm{s}$, $\mathrm{NH}), 13.15(1 \mathrm{H}, \mathrm{s}, \mathrm{SH})$.

4-((4,6-Dichloro-1,3,5-triazin-2-yl)amino )-5-methyl-4H-1,2,4triazole-3-thiol (7b)

From cyanuric chloride $(3.68 \mathrm{~g}, 20 \mathrm{mmol})$, potassium carbonate (2.76 g, $20 \mathrm{mmol}$ ) and compound (2b) (2.6 g, $20 \mathrm{mmol})$, compound (7b) was obtained as light yellow solid with yield of $(1.85 \mathrm{~g}, 33.25 \%)$ after $36 \mathrm{~h}$, melting point $\left(190-192{ }^{\circ} \mathrm{C}\right),{ }^{1} \mathrm{H}-\mathrm{NMR}$ $\left(\mathrm{DMSO}_{6}, \mathrm{ppm}\right) \delta: 2.16\left(3 \mathrm{H}, \mathrm{s}, \mathrm{CH}_{3}\right), 8.49(1 \mathrm{H}, \mathrm{s}, \mathrm{NH}), 13.31$ (1H, s, SH).

4-((4,6-Dichloro-1,3,5-triazin-2-yl) amino)-5-ethyl-4H-1,2,4triazole-3-thiol (7c)

From cyanuric chloride $(3.68 \mathrm{~g}, 20 \mathrm{mmol})$, potassium carbonate $(2.76 \mathrm{~g}, 20 \mathrm{mmol})$ and compound (2c) $(2.88 \mathrm{~g}, 20$ $\mathrm{mmol})$, compound $(7 \mathrm{c})$ was obtained as light yellow solid with yield of $(1.95 \mathrm{~g}, 33.4 \%)$ after $48 \mathrm{~h}$, melting point $\left(186-188^{\circ} \mathrm{C}\right),{ }^{1} \mathrm{H}-$ NMR (DMSO-d $\left.\mathrm{d}_{6}, \mathrm{ppm}\right) \delta: 1.34\left(3 \mathrm{H}, \mathrm{t}, J=7.0 \mathrm{~Hz}, \mathrm{CH}_{3}\right), 2.58(2 \mathrm{H}$, q, $\left.J=7.0 \mathrm{~Hz}, \mathrm{CH}_{2}\right), 8.25(1 \mathrm{H}, \mathrm{s}, \mathrm{NH}), 12.97(1 \mathrm{H}, \mathrm{s}, \mathrm{SH})$.

\section{5-Butyl-4-((4,6-dichloro-1,3,5-triazin-2-yl)amino)- 4H-1,2,4- triazole-3-thiol (7d)}

From cyanuric chloride (3.68 g, $20 \mathrm{mmol})$, potassium carbonate $(2.76 \mathrm{~g}, 20 \mathrm{mmol})$ and compound (2d) $(3.44 \mathrm{~g}, 20$ $\mathrm{mmol})$, compound (7d) was obtained as light yellow solid with 
yield of $(2.1 \mathrm{~g}, 33 \%)$ after $24 \mathrm{~h}$, melting boiling $\left(112-114^{\circ} \mathrm{C}\right),{ }^{1} \mathrm{H}$ NMR (DMSO-d $\left.\mathrm{d}_{6}, \mathrm{ppm}\right) \delta$ : $0.98\left(3 \mathrm{H}, \mathrm{t}, J=6.6 \mathrm{~Hz}, \mathrm{CH}_{3}\right), 1.39-1.42$ $\left(2 \mathrm{H}, \mathrm{m}, \mathrm{CH}_{3} \mathbf{C H}_{2}\right), 1.65-1.69$ (2H, m, $\left.\mathrm{CH}_{3} \mathrm{CH}_{2} \mathbf{C H}_{2}\right), 2.77(2 \mathrm{H}, \mathrm{t}$, $\left.J=5.8 \mathrm{~Hz}, \mathrm{CH}_{3} \mathrm{CH}_{2} \mathrm{CH}_{2} \mathbf{C H}_{2}\right), 8.85(1 \mathrm{H}, \mathrm{s}, \mathrm{NH}), 13.31(1 \mathrm{H}, \mathrm{s}, \mathrm{SH})$.

\section{4-((4,6-Dichloro-1,3,5-triazin-2-yl)amino)-5-pheny l-4H-1,2,4- triazole-3-thiol (7e)}

From cyanuric chloride $(3.68 \mathrm{~g}, 20 \mathrm{mmol})$, potassium carbonate $(2.76 \mathrm{~g}, 20 \mathrm{mmol})$ and compound (2e) $(3.8 \mathrm{~g}, 20 \mathrm{mmol})$, compound (7e) was obtained as off white solid in the yield of $(2.27 \mathrm{~g}, 33 \%)$ after $48 \mathrm{~h}$, melting point $\left(252-254^{\circ} \mathrm{C}\right),{ }^{1} \mathrm{H}-\mathrm{NMR}$ (DMSO-d6, ppm) $\delta: 7.35-7.39$ (1H, m, Ph 4-H), 7.45 (2H, t, $J=7.8$ $\left.\mathrm{Hz}, \mathrm{Ph} 3,5-\mathrm{H}_{2}\right), 7.68\left(2 \mathrm{H}, \mathrm{dd}, J=7.8,1.8 \mathrm{~Hz}, \mathrm{Ph} 2,6-\mathrm{H}_{2}\right), 8.94(1 \mathrm{H}$, s, NH), 13.39 (1H, s, SH).

\section{General procedures for synthesis of compounds $8 a-8 e$}

Excess ammonium hydroxide solution $(25 \mathrm{ml}, 28 \%)$ was added to $(3 \mathrm{mmol})$ of the corresponding thiazole derivative $(6 \mathrm{a}-6 \mathrm{e})$ and $(0.82 \mathrm{~g}, 6 \mathrm{mmol})$ potassium carbonate. The reaction mixture was heated under reflux for $72 \mathrm{~h}$. The reaction mixture was partitioned between methylene chloride $(250 \mathrm{ml})$ and $\mathrm{HCl}(0.1 \mathrm{M}$, $500 \mathrm{ml})$. The collected organic layer was washed with dist. water $(3 \times 75 \mathrm{ml})$, brine solution $(50 \mathrm{ml})$, dried over anhydrous magnesium sulfate and then evaporated under vacuum to obtain the pure product. Crystallization from aqueous ethanol is carried out for more purification.

\section{$N^{2}$-(4-phenylthiazol-2-yl)-1,3,5-triazine-2,4,6-triamine (8a)}

From excess ammonium hydroxide solution $(25 \mathrm{ml}$, $28 \%$ ), compound (6a) (0.97 g, $3 \mathrm{mmol})$ and potassium carbonate (0.82 $\mathrm{g}, 6 \mathrm{mmol})$, compound (8a) was obtained as light orange solidin the yield of $(0.27 \mathrm{~g}, 32 \%)$, melting point $\left(205-207^{\circ} \mathrm{C}\right) .{ }^{1} \mathrm{H}$ NMR (DMSO-d 6 , ppm) $\delta: 2.81\left(4 \mathrm{H}, \mathrm{s}, 2 \times \mathrm{NH}_{2}\right), 6.96(1 \mathrm{H}, \mathrm{s}, \mathrm{CH}$ of thiazole ring), $7.37(1 \mathrm{H}, \mathrm{m}, \mathrm{Ph} 4-\mathrm{H}), 7.47(2 \mathrm{H}, \mathrm{t}, J=7.6 \mathrm{~Hz}, \mathrm{Ph}$ 3,5- $\left.\mathrm{H}_{2}\right), 7.72\left(2 \mathrm{H}, \mathrm{d}, J=7.6 \mathrm{~Hz}, \mathrm{Ph} 2,6-\mathrm{H}_{2}\right), 8.48$ (1H, s, NH).Anal. Cald for $\mathrm{C}_{12} \mathrm{H}_{11} \mathrm{~N}_{7} \mathrm{~S}$ : C, 50.51; H, 3.89; N, 34.36; S, 11.24. Found: C, 50.43; H, 4.01; N, 34.55; S, 11.03 .

\section{$N^{2}$-(4-(4-chlorophenyl)thiazol-2-yl)-1,3,5-triazine-2,4,6 -triamine} (8b)

From excess ammonium hydroxide solution $(25 \mathrm{ml}$, $28 \%$ ), compound (6b) (1.07 g, $3 \mathrm{mmol})$ and potassium carbonate (0.82 g, $6 \mathrm{mmol})$, compound (8b) was obtained as off white solid in the yield of $(0.26 \mathrm{~g}, 28 \%)$, melting point $\left(245-247^{\circ} \mathrm{C}\right) .{ }^{1} \mathrm{H}-\mathrm{NMR}$ $\left(\right.$ DMSO-d $\left._{6}, \mathrm{ppm}\right) \delta: 2.65(4 \mathrm{H}, \mathrm{s}, 2 \mathrm{x} \mathrm{NH}), 7.26(1 \mathrm{H}, \mathrm{s}, \mathrm{CH}$ of

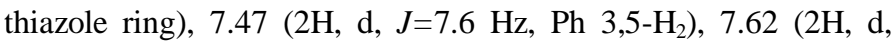
$J=7.6 \mathrm{~Hz}, \quad \mathrm{Ph}$ 2,6- $\left.\mathrm{H}_{2}\right), \quad 8.49(1 \mathrm{H}, \quad \mathrm{s}, \mathrm{NH})$. Anal. Calcd for $\mathrm{C}_{12} \mathrm{H}_{10} \mathrm{ClN}_{7} \mathrm{~S}$ : C, 45.07; H, 3.15; N, 30.66; S, 10.03. Found: C, 44.84; H, 3.29; N, 30.45; S, 10.15 .

\section{$N^{2}$-(4-(4-methoxyphenyl) thiazol-2-yl)-1,3,5-triazine-2,4,6- triamine $(8 c)$}

From excess ammonium hydroxide solution $(25 \mathrm{ml}$, $28 \%)$, compound (6c) (1.06 g, $3 \mathrm{mmol})$ and potassium carbonate $(0.82 \mathrm{~g}, 6 \mathrm{mmol})$, compound $(8 \mathrm{c})$ was obtained as light yellow solid in the yield of $(0.28 \mathrm{~g}, 30 \%)$, melting point (203$\left.205^{\circ} \mathrm{C}\right) .{ }^{1} \mathrm{H}-\mathrm{NMR}\left(\mathrm{DMSO}_{6}, \mathrm{ppm}\right) \delta: 3.49\left(4 \mathrm{H}, \mathrm{s}, 2 \times \mathrm{NH}_{2}\right), 3.78$ $\left(3 \mathrm{H}, \mathrm{s}, \mathrm{OCH}_{3}\right), 7.05\left(2 \mathrm{H}, \mathrm{d}, J=7.6 \mathrm{~Hz}, \mathrm{Ph} 3,5-\mathrm{H}_{2}\right), 7.12(1 \mathrm{H}, \mathrm{s}, \mathrm{CH}$ of thiazole ring), $7.63\left(2 \mathrm{H}, \mathrm{d}, J=7.6 \mathrm{~Hz}, \mathrm{Ph} 2,6-\mathrm{H}_{2}\right), 8.80(1 \mathrm{H}, \mathrm{s}$, $\mathrm{NH})$.Anal. Calcd for $\mathrm{C}_{13} \mathrm{H}_{13} \mathrm{~N}_{7} \mathrm{OS}$ : C, 49.51; H, 4.16; N, 31.09; S, 10.17. Found: C, 49.67; H, 4.38; N, 30.87; S, 10.03 .

\section{$N^{2}$-(4-(p-tolyl)thiazol-2-yl)-1,3,5-triazine-2,4,6-triamine (8d)}

From excess ammonium hydroxide solution $(25 \mathrm{ml}$, $28 \%$ ), compound (6d) (1.01 g, $3 \mathrm{mmol})$ and potassium carbonate $(0.82 \mathrm{~g}, 6 \mathrm{mmol})$, compound (8d) was obtained as white solid in the yield of $(0.26 \mathrm{~g}, 30 \%)$, melting point $\left(146-148^{\circ} \mathrm{C}\right) .{ }^{1} \mathrm{H}-\mathrm{NMR}$ (DMSO-d 6 , ppm) $\delta: 2.32\left(3 \mathrm{H}, \mathrm{s}, \mathrm{CH}_{3}\right), 2.79\left(4 \mathrm{H}, \mathrm{s}, 2 \mathrm{x} \mathrm{NH}_{2}\right), 7.13$

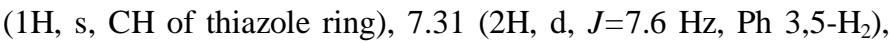
$7.45(1 \mathrm{H}, \mathrm{s}, \mathrm{NH}), 7.62\left(2 \mathrm{H}, \mathrm{d}, J=7.6 \mathrm{~Hz}, \mathrm{Ph} 2,6-\mathrm{H}_{2}\right)$.Anal. Calcd for $\mathrm{C}_{13} \mathrm{H}_{13} \mathrm{~N}_{7} \mathrm{~S}$ : C, 52.16; H, 4.38; N, 32.75; S, 10.71. Found: C, $51.98 ; \mathrm{H}, 4.54 ; \mathrm{N}, 32.53 ; \mathrm{S}, 10.92$.

\section{$N^{2}$-(4-(4-nitrophenyl)thiazol-2-yl)-1,3,5-triazine-2,4,6-triamine} $(8 e)$

From excess ammonium hydroxide solution $(25 \mathrm{ml}$, $28 \%)$, compound (6e) $(1.10 \mathrm{~g}, 3 \mathrm{mmol})$ and potassium carbonate (0.82 g, $6 \mathrm{mmol})$, compound (8e) was obtained as dark yellow solid in the yield of $(0.29 \mathrm{~g}, 30.2 \%)$, melting point $\left(227-229^{\circ} \mathrm{C}\right)$. ${ }^{1} \mathrm{H}-\mathrm{NMR}$ (DMSO-d 6 , ppm) $\delta: 3.52\left(4 \mathrm{H}, \mathrm{s}, 2 \times \mathrm{NH}_{2}\right), 7.32(1 \mathrm{H}, \mathrm{s}$, $\mathrm{CH}$ of thiazole ring), $7.93\left(2 \mathrm{H}, \mathrm{d}, J=7.6 \mathrm{~Hz}, \mathrm{Ph} 2,6-\mathrm{H}_{2}\right), 8.36(2 \mathrm{H}$, d, J=7.6 Hz, Ph 3,5- $\left.\mathrm{H}_{2}\right), 8.98(1 \mathrm{H}$, s, NH).Anal.Calcd for $\mathrm{C}_{12} \mathrm{H}_{10} \mathrm{~N}_{8} \mathrm{O}_{2} \mathrm{~S}$ : C, 43.63; H, 3.05; N, 33.92; S, 9.71. Found: C, 43.87; H, 2.82; N, 33.77; S, 9.65.

\section{General procedure for synthesis of compounds 9a-e}

Excess cyclohexylamine $(2.3 \mathrm{ml}, 20 \mathrm{mmol})$ was added to a suspension of $(3 \mathrm{mmol})$ of the corresponding triazole derivative $(6 a-6 e)$ and $(6 \mathrm{mmol})$ potassium carbonate in tetrahydrofuran. The reaction mixture was heated under reflux for $72 \mathrm{~h}$. The solvent was evaporated under vacuum and a dark brown viscous residue was obtained for all compounds except compound 10e which gave solid mass.

The residue was suspended in methylene chloride and was partitioned between methylene chloride $(250 \mathrm{ml})$ and $\mathrm{HCl}(0.1$ $\mathrm{M}, 500 \mathrm{ml})$.

The collected organic layer was washed with dist. water $(3 \times 75 \mathrm{ml})$, brine solution $(50 \mathrm{ml})$, dried over anhydrous magnesium sulfate and then concentrated under vacuum. The desired compound was precipitated from the organic solution by adding acetonitrile drop by drop till no more solid was precipitated. The precipitated solid was then filtered and purified by crystallization from aqueous ethanol.

\section{$N^{2}, N^{4}$-dicyclohexyl- $N^{6}$-(4-phenylthiazol-2-yl)-1,3,5-triazine- 2,4,6-triamine (9a)}

From excess cyclohexylamine $(2.3 \mathrm{ml}, 20 \mathrm{mmol})$, compound (6a) $(0.97 \mathrm{~g}, 3 \mathrm{mmol})$ and potassium carbonate $(0.82 \mathrm{~g}$, 
$6 \mathrm{mmol}$ ), compound (9a) was obtained as brown solid in the yield of $(0.39 \mathrm{~g}, 28.1 \%)$, melting point $\left(213-215^{\circ} \mathrm{C}\right) .{ }^{1} \mathrm{H}-\mathrm{NMR}$ (DMSO$\left.\mathrm{d}_{6}, \mathrm{ppm}\right) \delta: 1.2-1.7\left(20 \mathrm{H}, \mathrm{m}, 2 \mathrm{x}\right.$ cyclohexyl- $\left.\mathrm{H}_{10}\right), 3.24(2 \mathrm{H}, \mathrm{s}, 2 \mathrm{x}$ $\mathrm{NH}), 3.38-3.46(2 \mathrm{H}, \mathrm{m}, 2 \mathrm{x}$ cyclohexyl-H), $6.75(1 \mathrm{H}, \mathrm{s}, \mathrm{CH}$ of thiazole ring), 7.35-7.38 $(1 \mathrm{H}, \mathrm{m}, \mathrm{Ph} 4-\mathrm{H}), 7.44(2 \mathrm{H}, \mathrm{t}, J=7.6 \mathrm{~Hz}$, $\left.\mathrm{Ph} 3,5-\mathrm{H}_{2}\right), 7.67$ (2H, dd, J=7.6, 1.6 Hz, Ph 2,4-H $), 8.44(1 \mathrm{H}, \mathrm{s}$, $\mathrm{NH}$ ).Anal.Calcd for $\mathrm{C}_{24} \mathrm{H}_{31} \mathrm{~N}_{7} \mathrm{~S}$ : C, 64.11; H, 6.95; N, 21.81; S, 7.13. Found: C, 64.22; H, 7.28; N, 21.64; S, 6.95.

\section{$N^{2}$-(4-(4-chlorophenyl)thiazol-2-yl)- $N^{4}, N^{6}$ - dicyclohexyl-1,3,5- triazine-2,4,6-triamine $(9 \mathrm{~b})$}

From excess cyclohexylamine $(2.3 \mathrm{ml}, 20 \mathrm{mmol})$, compound (6b) $(1.07 \mathrm{~g}, 3 \mathrm{mmol})$ and potassium carbonate $(0.82 \mathrm{~g}$, $6 \mathrm{mmol}$ ), compound (9b) was obtained as buff solid in the yield of $(0.36 \mathrm{~g}, 25 \%)$, melting point $\left(219-221^{\circ} \mathrm{C}\right) .{ }^{1} \mathrm{H}-\mathrm{NMR}$ (DMSO-d $\mathrm{d}_{6}$, ppm) $\delta: 1.39-1.71\left(20 \mathrm{H}, \mathrm{m}, 2 \mathrm{x}\right.$ cyclohexyl- $\left.\mathrm{H}_{10}\right), 3.17-3.24(2 \mathrm{H}, \mathrm{m}$, $2 \mathrm{x}$ cyclohexyl-H), $3.86(2 \mathrm{H}, \mathrm{s}, 2 \mathrm{x} \mathrm{NH}), 6.81(1 \mathrm{H}, \mathrm{s}, \mathrm{CH}$ of thiazole ring), $7.47\left(2 \mathrm{H}, \mathrm{d}, J=7.6 \mathrm{~Hz}, \mathrm{Ph} 3,5-\mathrm{H}_{2}\right), 7.67(2 \mathrm{H}, \mathrm{d}, J=$ $7.6 \mathrm{~Hz}, \mathrm{Ph}$ 2,6- $\left.\mathrm{H}_{2}\right), 8.72(1 \mathrm{H}, \quad \mathrm{s}, \quad \mathrm{NH})$.Anal. Calcd for $\mathrm{C}_{24} \mathrm{H}_{30} \mathrm{CLN}_{7} \mathrm{~S}$ : C, 59.55; H, 6.25; N, 20.26; S, 6.62. Found: C, 59.30; H, 6.45; N, 20.33; S, 6.40 .

\section{$N^{2}, N^{4}$-dicyclohexyl- $N^{6}$-(4-(4-methoxyphenyl )thiazol-2-yl)-1,3,5- triazine-2,4,6-triamine $(9 c)$}

From excess cyclohexylamine $(2.3 \mathrm{ml}, 20 \mathrm{mmol})$, compound (6c) (1.06 g, $3 \mathrm{mmol})$ and potassium carbonate $(0.82 \mathrm{~g}$, $6 \mathrm{mmol}$ ), compound (9c) was obtained as buff solid in the yield of $(0.34 \mathrm{~g}, 24.1 \%)$, melting point $\left(154-156^{\circ} \mathrm{C}\right) .{ }^{1} \mathrm{H}-\mathrm{NMR}$ (DMSO-d ${ }_{6}$, ppm) $\delta: 1.25-1.74\left(20 \mathrm{H}, \mathrm{m}, 2 \mathrm{x}\right.$ cyclohexyl- $\left.\mathrm{H}_{10}\right), 3.22-3.29(2 \mathrm{H}, \mathrm{m}$, 2 x cyclohexyl-H), $3.78\left(3 \mathrm{H}, \mathrm{s}, \mathrm{OCH}_{3}\right), 3.79(2 \mathrm{H}, \mathrm{s}, \mathrm{NH}), 6.81$ $\left(1 \mathrm{H}, \mathrm{s}, \mathrm{CH}\right.$ of thiazole ring), $7.06\left(2 \mathrm{H}, \mathrm{d}, J=7.6 \mathrm{~Hz}, \mathrm{Ph} 3,5-\mathrm{H}_{2}\right)$, $7.63\left(2 \mathrm{H}, \mathrm{d}, J=7.6 \mathrm{~Hz}, \mathrm{Ph} 2,6-\mathrm{H}_{2}\right), 8.49(1 \mathrm{H}, \mathrm{s}, \mathrm{NH})$. Anal. Calcd for $\mathrm{C}_{25} \mathrm{H}_{33} \mathrm{~N}_{7} \mathrm{OS}$ : C, 62.60; H, 6.93; N, 20.44; S, 6.69.Found: C, 62.42; H, 6.65; N, 20.36; S, 6.87.

\section{$N^{2}, N^{4}$-dicyclohexyl- $N^{6}-(4-(p-t o l y l)$ thiazol-2-yl)-1,3,5-triazine- 2,4,6-triamine (9d)}

From excess cyclohexylamine $(2.3 \mathrm{ml}, 20 \mathrm{mmol})$, compound (6d) (1.01 g, $3 \mathrm{mmol})$ and potassium carbonate $(0.82 \mathrm{~g}$, $6 \mathrm{mmol}$ ), compound (9d) was obtained as buff solid in the yield of $(0.36 \mathrm{~g}, 25.9 \%)$, melting point $\left(128-129^{\circ} \mathrm{C}\right) .{ }^{1} \mathrm{H}-\mathrm{NMR}$ (DMSO-d ${ }_{6}$, ppm) $\delta: 1.29-1.79\left(20 \mathrm{H}, \mathrm{m}, 2 \mathrm{x}\right.$ cyclohexyl- $\left.\mathrm{H}_{10}\right), 2.32(3 \mathrm{H}, \mathrm{s}$, $\left.\mathrm{CH}_{3}\right), 3.15-3.23$ (2H, m, 2 x cyclohexyl-H), 3.34 (2H, s, 2 x NH), $6.83(1 \mathrm{H}, \mathrm{s}, \mathrm{CH}$ of thiazole ring $), 7.26(1 \mathrm{H}, \mathrm{s}, \mathrm{NH}), 7.31(2 \mathrm{H}, \mathrm{d}$, $\left.J=7.6 \mathrm{~Hz}, \mathrm{Ph} 3,5-\mathrm{H}_{2}\right), 7.61(2 \mathrm{H}, \mathrm{d}, J=7.6 \mathrm{~Hz}, \mathrm{Ph}$ 2,6$\mathrm{H}_{2}$ ).Anal.Calcd for $\mathrm{C}_{25} \mathrm{H}_{33} \mathrm{~N}_{7} \mathrm{~S}: \mathrm{C}, 64.76 ; \mathrm{H}, 7.17 ; \mathrm{N}, 21.15 ; \mathrm{S}$, 6.92. Found: C, 64.58; H,6.93; N,21.27; S,7.13.

\section{$N^{2}, N^{4}$-dicyclohexyl-N $N^{6}$-(4-(4-nitrophenyl)thiazol-2-yl)-1,3,5- triazine-2,4,6-triamine (9e)}

From excess cyclohexylamine $(2.3 \mathrm{ml}, 20 \mathrm{mmol})$, compound (6e) $(1.10 \mathrm{~g}, 3 \mathrm{mmol})$ and potassium carbonate $(0.82 \mathrm{~g}$, $6 \mathrm{mmol}$ ), compound (9e) was obtained as dark yellow solid in the yield of $(0.34 \mathrm{~g}, 23 \%)$, melting point $\left(201-202^{\circ} \mathrm{C}\right) .{ }^{1} \mathrm{H}-\mathrm{NMR}$ $\left(\right.$ DMSO-d $_{6}$, ppm) $\delta:$ 1.34-1.78 (20H, m, 2 x cyclohexyl- $\left.\mathrm{H}_{10}\right), 3.11-$ 3.19 (2H, m, 2 x cyclohexyl-H), 3.99 (2H, s, 2 x NH), $7.30(1 \mathrm{H}, \mathrm{s}$, $\mathrm{CH}$ of thiazole ring), $7.92\left(2 \mathrm{H}, \mathrm{d}, J=7.6 \mathrm{~Hz}, \mathrm{Ph} 2,6-\mathrm{H}_{2}\right), 8.36(2 \mathrm{H}$, d, J=7.6 Hz, $\left.\mathrm{Ph} 3,5-\mathrm{H}_{2}\right), 8.94(1 \mathrm{H}$, s, NH).Anal.Calcd for $\mathrm{C}_{24} \mathrm{H}_{30} \mathrm{~N}_{8} \mathrm{O}_{2} \mathrm{~S}$ : C, 58.28; H, 6.11; N, 22.65; S, 6.48. Found: C, 58.35; H, 5.93; N, 22.83; S, 6.67.

\section{General procedures for synthesis of compounds 10a-e}

Excess ammonium hydroxide solution $(25 \mathrm{ml}, 28 \%)$ was added to $(3 \mathrm{mmol})$ of the corresponding compound (7a-7e) and $(0.82 \mathrm{~g}, 6 \mathrm{mmol})$ potassium carbonate. The reaction mixture was heated under reflux for $(24-48 \mathrm{~h})$. The reaction mixture was partitioned between methylene chloride $(250 \mathrm{ml})$ and and $\mathrm{HCl}(0.1$ M, $500 \mathrm{ml})$. The collected organic layer was washed with dist. water $(3 \times 75 \mathrm{ml})$, brine solution $(50 \mathrm{ml})$, dried over magnesium sulfate and then evaporated under vacuum to obtain the pure product of $10 \mathrm{a}, 10 \mathrm{c}$ and $10 \mathrm{e}$. In case of $10 \mathrm{~b}$ and $10 \mathrm{~d}$; evaporation of organic layer gives viscous residue which was dissolved in acetone. The acetone solution was left overnight in refrigerator and the precipitated solid product was then filtered and dried. Crystallization using aqueous ethanol was carried out for purification.

\section{4-((4,6-Diamino-1,3,5-triazin-2-yl) amino)-4H-1,2,4-triazole-3- thiol (10a)}

From excess ammonium hydroxide solution $(25 \mathrm{ml}$, $28 \%$ ), compound (7a) (0.79 g, $3 \mathrm{mmol})$ and potassium carbonate $(0.82 \mathrm{~g}, 6 \mathrm{mmol})$, compound (10a) was obtained as off-white solid in the yield of $(0.271 \mathrm{~g}, 40.1 \%)$ after $48 \mathrm{~h}$, melting point (141$\left.143^{\circ} \mathrm{C}\right) .{ }^{1} \mathrm{H}-\mathrm{NMR}\left(\mathrm{DMSO}_{6}, \mathrm{ppm}\right) \delta: 2.73\left(4 \mathrm{H}, \mathrm{s}, 2 \mathrm{x} \mathrm{NH}_{2}\right), 8.120$ $(1 \mathrm{H}, \mathrm{s}, \mathrm{H}$ on triazole ring), 8.91 $(1 \mathrm{H}, \mathrm{s}, \mathrm{NH}), 13.26(1 \mathrm{H}, \mathrm{s}$, $\mathrm{SH})$.Anal. Calcd for $\mathrm{C}_{5} \mathrm{H}_{7} \mathrm{~N}_{9} \mathrm{~S}: \mathrm{C}, 26.66 ; \mathrm{H}, 3.13 ; \mathrm{N}, 55.97 ; \mathrm{S}$, 14.24. Found: C, 26.89; H, 3.35; N, 55.88; S, 13.98.

\section{4-((4,6-Diamino-1,3,5-triazin-2-yl )amino)-5-methyl-4H-1,2,4- triazole-3-thiol (10b)}

From excess ammonium hydroxide solution $(25 \mathrm{ml}$, $28 \%$ ), compound (7b) (0.83 g, $3 \mathrm{mmol})$ and potassium carbonate $(0.82 \mathrm{~g}, 6 \mathrm{mmol})$, compound $(10 \mathrm{~b})$ was obtained as buff solid in the yield of $(0.26 \mathrm{~g}, 36.5 \%)$ after $36 \mathrm{~h}$, melting point $\left(114-116^{\circ} \mathrm{C}\right) .{ }^{1} \mathrm{H}$ NMR (DMSO-d 6 , ppm) $\delta: 2.29\left(3 \mathrm{H}, \mathrm{s}, \mathrm{CH}_{3}\right), 2.82(4 \mathrm{H}, \mathrm{s}, 2 \mathrm{x}$ $\left.\mathrm{NH}_{2}\right), 8.70(1 \mathrm{H}, \mathrm{s}, \mathrm{NH}), 12.93(1 \mathrm{H}, \mathrm{s}, \mathrm{SH})$. Anal.Calcd for $\mathrm{C}_{6} \mathrm{H}_{9} \mathrm{~N}_{9} \mathrm{~S}: \mathrm{C}, 30.12 ; \mathrm{H}, 3.79 ; \mathrm{N}, 52.69 ; \mathrm{S}, 13.40$. Found: C,30.23; H, 3.88; N, 52.45; S, 13.56 .

\section{4-((4,6-diamino-1,3,5-triazin-2-yl)amino)-5-ethyl-4H-1,2,4- triazole-3-thiol (10c)}

From excess ammonium hydroxide solution $(25 \mathrm{ml}$, $28 \%)$, compound $(7 \mathrm{c})(0.87 \mathrm{~g}, 3 \mathrm{mmol})$ and potassium carbonate $(0.82 \mathrm{~g}, 6 \mathrm{mmol})$, compound (10c) was obtained as light rose solid in the yield of $(0.25 \mathrm{~g}, 32.9 \%)$ after $48 \mathrm{~h}$, melting point (208$209^{\circ} \mathrm{C}$ ). ${ }^{1} \mathrm{H}-\mathrm{NMR}$ (DMSO-d 6 , ppm) $\delta: 1.31\left(3 \mathrm{H}, \mathrm{t}, J=6.8 \mathrm{~Hz}, \mathrm{CH}_{3}\right.$ ), $2.58\left(2 \mathrm{H}, \mathrm{d}, J=6.8 \mathrm{~Hz}, \mathrm{CH}_{2}\right), 2.94\left(4 \mathrm{H}, \mathrm{s}, 2 \mathrm{x} \mathrm{NH}_{2}\right), 8.44(1 \mathrm{H}, \mathrm{s}$, 
$\mathrm{NH}), 13.17(1 \mathrm{H}, \mathrm{s}, \mathrm{SH})$.Anal.Calcd for $\mathrm{C}_{7} \mathrm{H}_{11} \mathrm{~N}_{9} \mathrm{~S}$ : C, 33.19; $\mathrm{H}, 4.38$; N, 49.77; S, 12.66. Found: C, 33.34; H, 4.15; N, 49.56; S, 12.93.

\section{5-Butyl-4-((4,6-diamino-1,3,5-triazin-2-yl) amino)-4H-1,2,4- triazole-3-thiol (10d)}

From excess ammonium hydroxide solution $(25 \mathrm{ml}$, $28 \%$ ), compound (7d) (0.96 g, $3 \mathrm{mmol})$ and potassium carbonate (0.82 g, $6 \mathrm{mmol})$, compound (10d) was obtained as reddish brownsolid in the yield of $(0.27 \mathrm{~g}, 32.9 \%)$ after $24 \mathrm{~h}$, melting point $\left(229-230^{\circ} \mathrm{C}\right) .{ }^{1} \mathrm{H}-\mathrm{NMR}\left(\mathrm{DMSO}-\mathrm{d}_{6}, \mathrm{ppm}\right) \delta: 0.99(3 \mathrm{H}, \mathrm{t}, J=6.8 \mathrm{~Hz}$, $\left.\mathrm{CH}_{3}\right), \quad 1.38-1.43 \quad\left(2 \mathrm{H}, \quad \mathrm{m}, \quad \mathrm{CH}_{3} \mathbf{C H}_{2}\right), \quad 1.58-1.61 \quad(2 \mathrm{H}, \quad \mathrm{m}$, $\left.\mathrm{CH}_{3} \mathrm{CH}_{2} \mathbf{C H}_{2}\right), 2.81\left(2 \mathrm{H}, \mathrm{t}, J=6.0 \mathrm{~Hz}, \mathrm{CH}_{3} \mathrm{CH}_{2} \mathrm{CH}_{2} \mathbf{C H}_{2}\right), 3.02(4 \mathrm{H}$, s, $\left.2 \times \mathrm{NH}_{2}\right), 8.88(1 \mathrm{H}, \mathrm{s}, \mathrm{NH}), 13.33(1 \mathrm{H}, \mathrm{s}, \mathrm{SH})$.Anal.Calcd for $\mathrm{C}_{9} \mathrm{H}_{15} \mathrm{~N}_{9} \mathrm{~S}$ : C, 38.42; H, 5.37; N, 44.81; S, 11.40. Found: C, 38.15; H, 5.54; N, 44.67; S, 11.63.

\section{4-((4,6-Diamino-1,3,5-triazin-2-yl)amino) -5-phenyl-4H-1,2,4- triazole-3-thiol (10e)}

From excess ammonium hydroxide solution $(25 \mathrm{ml}$, $28 \%)$, compound (7e) $(1.02 \mathrm{~g}, 3 \mathrm{mmol})$ and potassium carbonate $(0.82 \mathrm{~g}, 6 \mathrm{mmol})$, compound $(10 \mathrm{e})$ was obtained as buff solid in the yield of $(0.29 \mathrm{~g}, 33 \%)$ after $48 \mathrm{~h}$, melting point $\left(271-273^{\circ} \mathrm{C}\right) .{ }^{1} \mathrm{H}$ NMR (DMSO-d $\left.{ }_{6}, \mathrm{ppm}\right) \delta: 2.81\left(4 \mathrm{H}, \mathrm{s}, 2 \mathrm{x} \mathrm{NH}_{2}\right), 7.34-7.39(1 \mathrm{H}, \mathrm{m}$,

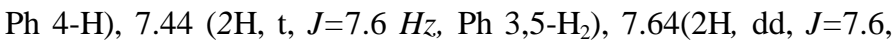
$\left.1.6 \mathrm{~Hz}, \mathrm{Ph} 2,6-\mathrm{H}_{2}\right), 8.97 \quad(1 \mathrm{H}, \quad \mathrm{s}, \mathrm{NH}), 13.41(1 \mathrm{H}, \mathrm{s}$, $\mathrm{SH})$.Anal.Calcd for $\mathrm{C}_{11} \mathrm{H}_{11} \mathrm{~N}_{9} \mathrm{~S}: \mathrm{C}, 43.84 ; \mathrm{H}, 3.68 ; \mathrm{N}, 41.83 ; \mathrm{S}$, 10.64. Found: C, 44.01; H, 3.43; N, 42.07; S, 10.48 .

\section{General procedures for synthesis of compounds 11a-11e}

Excess cyclohexylamine $(2.3 \mathrm{ml}, 20 \mathrm{mmol})$ was added to a suspension of ( $3 \mathrm{mmol})$ of the corresponding compound (7a-7e) and $(6 \mathrm{mmol})$ potassium carbonate in tetrahydrofuran. The reaction mixture was heated under reflux for (24-48) $\mathrm{h}$. The solvent was concentrated under vacuum and the resulted dark brown viscous residue was suspended in methylene chloride. The organic solution was partitioned between methylene chloride $(250 \mathrm{ml})$ and $\mathrm{HCl}(0.1 \mathrm{M}, 500 \mathrm{ml})$. The organic layer was washed with dist. water $(3 \times 75 \mathrm{ml})$, brine solution $(50 \mathrm{ml})$, dried over anhydrous magnesium sulfate and then concentrated under vacuum. The product was precipitated from organic brown solution by adding acetonitrile drop by drop till no more solid was formed. The precipitated solid was separated by filtration and purified by crystallization from aqueous ethanol.

\section{4-((4,6-Bis(cyclohexylamino)-1,3,5-triazin-2-yl)amino)-4H-1,2,4- triazole-3-thiol (11a)}

From excess cyclohexylamine $(2.3 \mathrm{ml}, 20 \mathrm{mmol})$, compound (7a) $(0.79 \mathrm{~g}, 3 \mathrm{mmol})$ and potassium carbonate $(0.82 \mathrm{~g}$, $6 \mathrm{mmol}$ ), compound (11a) was obtained as buff solid in the yield of $(0.35 \mathrm{~g}, 30.1 \%)$ after $48 \mathrm{~h}$, melting point $\left(165-167^{\circ} \mathrm{C}\right) .{ }^{1} \mathrm{H}-\mathrm{NMR}$ $\left(\right.$ DMSO-d $\left._{6}, \mathrm{ppm}\right) \delta: 1.35-1.73\left(20 \mathrm{H}, \mathrm{m}, 2 \mathrm{x}\right.$ cyclohexyl- $\left.\mathrm{H}_{10}\right), 3.33$ $(2 \mathrm{H}, \mathrm{s}, \mathrm{NH}), 3.60-3.67(2 \mathrm{H}, \mathrm{m}, 2 \mathrm{x}$ cyclohexyl-H), $8.08(1 \mathrm{H}, \mathrm{s}, \mathrm{CH}$ of triazole ring), $8.90(1 \mathrm{H}, \mathrm{s}, \mathrm{NH}), 13.12(1 \mathrm{H}, \mathrm{s}, \mathrm{SH})$. Anal. Calcd for $\mathrm{C}_{17} \mathrm{H}_{27} \mathrm{~N}_{9} \mathrm{~S}$ : C, 52.42; H, 6.99; N, 32.36; S, 8.23. Found:C, $52.21 ; \mathrm{H}, 7.26 ; \mathrm{N}, 32.14 ; \mathrm{S}, 8.55$.

\section{4-((4,6-bis(cyclohexylamino)-1,3,5-triazin-2-yl)amino)-5-methyl- 4H-1,2,4-triazole-3-thiol (11b)}

From excess cyclohexylamine $(2.3 \mathrm{ml}, 20 \mathrm{mmol})$, compound (7b) (0.83 g, $3 \mathrm{mmol})$ and potassium carbonate $(0.82 \mathrm{~g}$, $6 \mathrm{mmol}$ ), compound (11b) was obtained as buff solid in the yield of $(0.36 \mathrm{~g}, 30.1 \%)$ after $36 \mathrm{~h}$, melting point $\left(133-135^{\circ} \mathrm{C}\right) .{ }^{1} \mathrm{H}-\mathrm{NMR}$ $\left(\right.$ DMSO-d $\left._{6}, \mathrm{ppm}\right) \delta: 1.35-1.68\left(20 \mathrm{H}, \mathrm{m}, 2 \mathrm{x}\right.$ cyclohexyl- $\left.\mathrm{H}_{10}\right), 2.26$ (3H, s, $\left.\mathrm{CH}_{3}\right), 3.21-3.31$ (2H, m, 2 x cyclohexyl-H), 3.37 (2H, s, 2 x $\mathrm{NH}), 8.95(1 \mathrm{H}, \mathrm{s}, \mathrm{NH}), 13.04(1 \mathrm{H}, \mathrm{s}, \mathrm{SH})$. Anal.Calcd for $\mathrm{C}_{18} \mathrm{H}_{29} \mathrm{~N}_{9} \mathrm{~S}$ : C, 53.57; H, 7.24; N, 31.24; S, 7.95. Found: C, 53.72; H, 7.11; N, 30.98; S, 8.13.

\section{4-((4,6-Bis(cyclohexylamino)- 1,3,5-triazin-2-yl)amino)-5-ethyl- 4H-1,2,4-triazole-3-thiol (11c)}

From excess cyclohexylamine $(2.3 \mathrm{ml}, 20 \mathrm{mmol})$, compound $(7 \mathrm{c})(0.87 \mathrm{~g}, 3 \mathrm{mmol})$ and potassium carbonate $(0.82 \mathrm{~g}$, $6 \mathrm{mmol}$ ), compound (11c) was obtained as buff solid in the yield of $(0.34 \mathrm{~g}, 27.4 \%)$ after $48 \mathrm{~h}$, melting point $\left(217-219^{\circ} \mathrm{C}\right) .{ }^{1} \mathrm{H}-\mathrm{NMR}$ $\left(\right.$ DMSO-d $\left._{6}, \mathrm{ppm}\right) \delta: 1.25-1.72\left(23 \mathrm{H}, \mathrm{m}, 2 \mathrm{x}\right.$ cyclohexyl- $\mathrm{H}_{10}+$ $\left.\mathrm{CH}_{3}\right), 2.59\left(2 \mathrm{H}, \mathrm{q}, J=6.8 \mathrm{~Hz}, \mathrm{CH}_{2}\right), 3.78-3.81(2 \mathrm{H}, \mathrm{m}, 2 \mathrm{x}$ cyclohexyl-H), $4.02(2 \mathrm{H}, \mathrm{s}, 2$ x NH), $8.85(1 \mathrm{H}, \mathrm{s}, \mathrm{NH}), 13.25(1 \mathrm{H}$, s, SH).Anal.Calcd for $\mathrm{C}_{19} \mathrm{H}_{31} \mathrm{~N}_{9} \mathrm{~S}: \mathrm{C}, 54.65 ; \mathrm{H}, 7.48 ; \mathrm{N}, 30.19 ; \mathrm{S}$, 7.68 Found: C, 54.32; H, 7.31; N, 30.46; S, 7.80.

\section{4-((4,6-Bis(cyclohexylamino)- 1,3,5-triazin-2-yl)amino)-5-butyl- 4H-1,2,4-triazole-3-thiol (11d)}

From excess cyclohexylamine $(2.3 \mathrm{ml}, 20 \mathrm{mmol})$, compound (7d) $(0.96 \mathrm{~g}, \quad 3 \mathrm{mmol})$ and potassium carbonate $(0.82 \mathrm{~g}, 6 \mathrm{mmol})$, compound (11d) was obtained as light brown solid in the yield of $(0.34 \mathrm{~g}, 25.9 \%)$ after $24 \mathrm{~h}$, melting point $\left(153-155^{\circ} \mathrm{C}\right) .{ }^{1} \mathrm{H}-\mathrm{NMR}$ (DMSO-d $\left.{ }_{6}, \mathrm{ppm}\right) \delta: 0.98-1.72(27 \mathrm{H}$, m, 2 x cyclohexyl- $\left.H_{10}+\mathbf{C H}_{3} \mathbf{C H}_{2} \mathbf{C H}_{2}\right), 2.75(2 \mathrm{H}, \mathrm{t}, J=5.8 \mathrm{~Hz}$, $\left.\mathrm{CH}_{2}\right), 3.23(2 \mathrm{H}, \mathrm{s}, 2 \mathrm{x} \mathrm{NH}), 3.79-3.82(2 \mathrm{H}, \mathrm{m}, 2 \mathrm{x}$ cyclohexyl-H), $8.96(1 \mathrm{H}, \mathrm{s}, \mathrm{NH}), 13.23(1 \mathrm{H}, \mathrm{s}, \mathrm{SH})$.Anal.Calcd for $\mathrm{C}_{21} \mathrm{H}_{35} \mathrm{~N}_{9} \mathrm{~S}: \mathrm{C}$, 56.60; H, 7.92; N, 28.29; S, 7.20. Found: C, 56.42; H, 8.04; N, 28.37; S, 7.17.

\section{4-((4,6-bis(cyclohexylamino)- 1,3,5-triazin-2-yl)amino)-5-phenyl- 4H-1,2,4-triazole-3-thiol (11e)}

From excess cyclohexylamine $(2.3 \mathrm{ml}, 20 \mathrm{mmol})$, compound (7e) (1.02 g, $3 \mathrm{mmol})$ and potassium carbonate $(0.82 \mathrm{~g}$, $6 \mathrm{mmol}$ ), compound (11e) was obtained as buff solid in the yield of $(0.35 \mathrm{~g}, 25.1 \%)$ after $48 \mathrm{~h}$, melting point $\left(167-169^{\circ} \mathrm{C}\right) .{ }^{1} \mathrm{H}-\mathrm{NMR}$ $\left(\right.$ DMSO-d $\left._{6}, \mathrm{ppm}\right) \delta: 1.32-1.66\left(20 \mathrm{H}, \mathrm{m}, 2 \mathrm{x}\right.$ cyclohexyl- $\left.\mathrm{H}_{10}\right), 3.30$ (2H, s, 2 x NH), 3.38-3.50 (2H, m, 2 x cyclohexyl-H), 7.34-7.39 (1H, m, Ph 4-H), 7.44 (2H, t, J = 7.6 Hz, Ph 3,5- $\left.\mathrm{H}_{2}\right), 7.65(2 \mathrm{H}, \mathrm{dd}$, $\left.J=7.6,1.6 \mathrm{~Hz}, \mathrm{Ph} 2,6-\mathrm{H}_{2}\right), 8.98(1 \mathrm{H}, \mathrm{s}, \mathrm{NH}), 13.36(1 \mathrm{H}, \mathrm{s}$, $\mathrm{SH}$.Anal.Calcd for $\mathrm{C}_{23} \mathrm{H}_{31} \mathrm{~N}_{9} \mathrm{~S}$ : C, 59.33; H, 6.71; N, 27.07; S, 6.89. Found: C, 59.13; H, 6.63; N, 27.25; S, 6.98. 


\section{4,6-dichloro-N-phenyl-1,3,5-triazin-2-amine (12)}

To an ice cooled suspension of $(1.84 \mathrm{~g}, 10 \mathrm{mmol})$ cyanuric chloride in methylene chloride $(50 \mathrm{ml})$, and $(1.38 \mathrm{~g}, 10$ $\mathrm{mmol})$ potassium carbonate, aniline $(0.93 \mathrm{~g}, 10 \mathrm{mmol})$ was added drop wise with stirring over a period of $2 \mathrm{~h}$. After $4 \mathrm{~h}$, the reaction mixture was partitioned between methylene chloride $(250 \mathrm{ml})$ and $\mathrm{HCl}(0.1 \mathrm{M}, 500 \mathrm{ml})$.

The collected organic layer was washed with dist. water $(3 \times 75 \mathrm{ml})$, brine solution $(50 \mathrm{ml})$, dried over anhydrous magnesium sulfate and then evaporated under vacuum to obtain the product as white solid in the yield of $(1.73 \mathrm{~g}, 71.7 \%)$, melting point $\left(134-135^{\circ} \mathrm{C}\right) \stackrel{37)}{ }{ }^{1} \mathrm{H}-\mathrm{NMR}\left(\mathrm{DMSO}-\mathrm{d}_{6}, \mathrm{ppm}\right) \delta: 6.96-7.01(1 \mathrm{H}$, m, Ph 4-H), 7.029 (2H, dd, J=7.8, $\left.1.4 \mathrm{~Hz}, \mathrm{Ph} 2,6-\mathrm{H}_{2}\right), 7.27$ (2H, t, $\left.J=7.8 \mathrm{~Hz}, \mathrm{Ph} 3,5-\mathrm{H}_{2}\right), 8.53(1 \mathrm{H}, \mathrm{s}, \mathrm{NH})$.

\section{$N^{2}$-phenyl-1,3,5-triazine-2,4,6-triamine (13)}

Excess ammonium hydroxide solution (25 ml, 28\%) was added to ( $0.72 \mathrm{~g}, 3 \mathrm{mmol})$ of compound (12). The reaction mixture was heated under reflux for $4 \mathrm{~h}$. A suspension of $(0.82 \mathrm{~g}, 6 \mathrm{mmol})$ potassium carbonate in water was added drop wise. The reaction mixture was partitioned between methylene chloride $(250 \mathrm{ml})$ and $\mathrm{HCl}(0.1 \mathrm{M}, 500 \mathrm{ml})$.

The collected organic layer was washed with dist. water $(3 \times 75 \mathrm{ml})$, brine solution $(50 \mathrm{ml})$, dried over magnesium sulfate and then evaporated under vacuum to obtain the product as white solid in the yield of $(0.48 \mathrm{~g}, 80.6 \%)$, melting point $\left(204-205^{\circ} \mathrm{C}\right)$. ${ }^{1} \mathrm{H}-\mathrm{NMR}\left(\mathrm{DMSO}_{-} \mathrm{d}_{6}, \mathrm{ppm}\right) \delta: 3.06\left(4 \mathrm{H}, \mathrm{s}, 2 \times \mathrm{NH}_{2}\right), 7.02(2 \mathrm{H}, \mathrm{dd}$, $\left.J=7.6,1.6 \mathrm{~Hz}, \mathrm{Ph} 2,6-\mathrm{H}_{2}\right), 7.08-7.13(1 \mathrm{H}, \mathrm{m}, \mathrm{Ph} 4-\mathrm{H}), 7.27$ (2H, t, $\left.J=7.6 \mathrm{~Hz}, \quad \mathrm{Ph} 3,5-\mathrm{H}_{2}\right), \quad 8.74 \quad(1 \mathrm{H}, \quad \mathrm{s}, \quad \mathrm{NH})$.Anal.Calcd.for $\mathrm{C}_{9} \mathrm{H}_{10} \mathrm{~N}_{6}$ : C, 53.46; H, 4.98; N, 41.56. Found: C, 53.35; H, 4.72;N, 41.79 .

\section{$N^{2}, N^{4}$-dicyclohexyl- $N^{6}$-phenyl-1,3,5-triazine-2,4,6-triamine (14)}

Excess cyclohexylamine $(2.3 \mathrm{ml}, 20 \mathrm{mmol})$ was added to

(0.72 g, $3 \mathrm{mmol})$ of compound (12) in tetrahydrofuran. The reaction mixture was heated under reflux for $4 \mathrm{~h}$. A suspension of $(0.82 \mathrm{~g}, 6 \mathrm{mmol})$ potassium carbonate in tetrahydrofuran was added drop wise. After $4 \mathrm{~h}$ the reaction mixture was partitioned between methylene chloride $(250 \mathrm{ml})$ and $\mathrm{HCl}(0.1 \mathrm{M}, 500 \mathrm{ml})$. The collected organic layer was washed with dist. water $(3 \times 75 \mathrm{ml})$, brine solution $(50 \mathrm{ml})$, dried over magnesium sulfate and then evaporated under vacuum to obtain the product as buff solid in the yield of $(0.66 \mathrm{~g}, 60 \%)$, melting point $\left(197-199^{\circ} \mathrm{C}\right) .{ }^{1} \mathrm{H}-\mathrm{NMR}$ (DMSO-d ${ }_{6}$, ppm) $\delta: 1.34-1.72\left(20 \mathrm{H}, \mathrm{m}, 2\right.$ x cyclohexyl-H $\left.\mathrm{H}_{10}\right), 3.32$ $(2 \mathrm{H}, \mathrm{s}, \mathrm{NH}), 3.70-3.77$ (2H, m, 2 x cyclohexyl-H ), 6.96-7.01 $(1 \mathrm{H}$, m, Ph 4-H), 7.02 (2H, dd, J=7.6, 1.4 Hz, Ph 2,6- $\left.\mathrm{H}_{2}\right), 7.27$ (2H, t, $\left.J=7.6 \mathrm{~Hz}, \mathrm{Ph} 3,5-\mathrm{H}_{2}\right), 9.03(1 \mathrm{H}, \mathrm{s}, \mathrm{NH})$.Anal.calcd. forC ${ }_{21} \mathrm{H}_{30} \mathrm{~N}_{6}$ : C, 68.82; H, 8.25; N, 22.93. Found: C, 69.15; H, 8.10; N, 22.71.

\section{Evaluation of cytotoxic activity against (A549) cancer cell line Cell line}

Human tumor carcinoma cell line (non-small cell lung carcinoma cell line (A549)) used in this study was supplied by the American Type Culture Collection (ATCC, Minisota, U.S.A.) and were maintained at the National Cancer Institute, Cairo, Egypt, by serial sub-culturing.

\section{Cytotoxic activity test}

A cryotube containing frozen cells (A549) was taken out of the liquid nitrogen container and then warmed in a water bath at $37^{\circ} \mathrm{C}$. The cryotube was opened under strict aseptic conditions and its contents were supplied by $5 \mathrm{ml}$ supplemented medium drop by drop in a $50 \mathrm{ml}$ sterile falcon tubes. The tube was incubated for $2 \mathrm{~h}$ then centrifuged at $1200 \mathrm{rpm}$ for $10 \mathrm{~min}$. The supernatant was discarded and the cell pellet was suspended and seeded in $5 \mathrm{ml}$ supplemented medium in T25 Nunclon sterile tissue culture flasks. The cells suspension was incubated and followed up daily. The supplemented medium was replaced every 2- 3 days. Incubation was continued until a confluent growth was achieved and the cells were freshly sub cultured before each experiment to be in the exponential phase of growth. The medium was discarded and the monolayer cell was washed twice with $5 \mathrm{ml}$ phosphate buffered saline. All the adherent cells were dispersed from their monolayer by the addition of $1 \mathrm{ml}$ trypsin solution $(0.25 \%$ trypsin $\mathrm{w} / \mathrm{v})$ for 2 min. $50 \mu 1$ of $0.05 \%$ trypan blue solution was added to $50 \mu \mathrm{l}$ of the single cell suspension. The cells were examined under the inverted microscope using the haemocytometer. Non stained (viable) cells were counted and the following equation was used to calculate the cell count $/ \mathrm{ml}$ of cell suspension.

Viable cells $/ \mathrm{ml}=$ $\underline{\text { number of cells in } 4 \text { quarters } X 2 \text { (dilution factor) } \times 10^{4}}$

4

The cells were then diluted to give the required cell number for each experiment.

The cytotoxicity was carried out using SulphorhodamineB (SRB) assay (Vichai and Kirtikara, 2006). Cells were seeded in 96-well microtiter plates at initial concentration of $3 \times 10^{3}$ cell/well in a $150 \mu \mathrm{l}$ fresh medium and left for 24 hours to attach to the plates. Different concentrations $0,5,12.5,25,50,100 \mu \mathrm{g} / \mathrm{ml}$ of tested compounds dissolved in DMSO were added. For each compound concentration, 3 wells were used. The plates were incubated for $48 \mathrm{~h}$. The cells were fixed with $50 \mu \mathrm{l}$ cold trichloroacetic acid $10 \%$ final concentration for $1 \mathrm{~h}$ at $4^{\circ} \mathrm{C}$. The plates were washed with distilled water and stained with $50 \mu \mathrm{l} 0.4$ $\%$ SRB dissolved in $1 \%$ acetic acid for 30 minutes at room temperature.

The plates were washed with $1 \%$ acetic acid and airdried.The dye was solubilized with $100 \mu \mathrm{l} /$ well of $10 \mathrm{M}$ tris base (pH 10.5) and optical density (O.D.) of each well was measured spectrophotometrically at $570 \mathrm{~nm}$ with an ELISA microplate reader (Sunrise Tecan reader, Germany). The mean background absorbance was automatically subtracted and means values of each tested compounds concentration was calculated. The experiment was repeated 3 times.

The percentage of cell survival was calculated as follows: Surviving fraction $=$ O.D. (treated cells)/ O.D. $($ control cells $)$. 
The $\mathrm{IC}_{50}$ values (the concentrations of tested compounds required to produce $50 \%$ inhibition of cell growth) were graphically determined from the concentration-response curves. Methotrexate was used as reference compound. A triplicate experiment was performed for each compound.

\section{Statistical analysis}

Data were statistically analyzed using SPSS 21.0 program (SPSS, Chicago, IL, USA) (IBM Software, 2015). The results are presented as mean \pm standard deviation (SD). Differences between compounds were evaluated statistically using one-way ANOVA (Tukey's test) after normality of distribution had been evaluated by Bartlett's test. The difference was considered significant when $\mathrm{P}$ value less than 0.05 .

\section{Molecular docking}

Molecular docking study was performed to investigate the binding affinities and interaction modes between the inhibitors and the target enzyme and correlate DHFR inhibition activities of the synthesized compounds to their chemical structures. Docking process requires a three dimensional (3D) structure of both protein and ligand. Structure-data file (MDL SD file) file format (*.sdf or *.sd) was used to save the three dimensional (3D) structures of all molecules that generated, cleaned and geometrically optimized using the ChemAxon ${ }^{\circledR}$ MarvinSketch 5.1.4 (ChemAxon, 2014) in order to be readable by FlexX (BioSolveIT, 2014). Library of methotrexate and newly synthesized target compounds was generated using Mona software (BioSolveIT GmbH, 2015). Several structures of hDHFR are available in protein data bank (PDB), ID: 4M6K (Protein data bank, 2015) was chosen for its good resolution $(1.40 \AA)$ and it bounds to its cofactor (NADP+) and its active ligand (folate).

The chemical model underlying FlexX is based on the work of Böhm (Böhm, 1994). The model can be divided into three areas: conformational flexibility, protein-ligand interactions, and the scoring function used for ranking the solutions generated. FlexX flexibly places ligands into the active site with an incremental buildup algorithm (pose clustering) (Rarey et al., 1996). It starts with selecting a base fragment, which is placed into the active site based on superposing interaction points of the fragment and the active site (pattern recognition technique). The base fragment is then incrementally built up to the complete compound by modeling the ligand flexibility with a torsion library for the added components (Pan et al., 2003). Placement of the ligand is scored based on protein-ligand interactions where, the binding energy for solutions generated is estimated, and placements are ranked (Van et al., 2007).

FlexX module defines active site amino acids in the target molecule during the target preparation step and can specify atoms belong to the binding site. Enzyme components such as $\mathrm{NADP}+$ cofactor is specified as a part of our protein which will occupy space in 3D during docking simulation. The active site for docking was determined as a sphere of all atoms within $8 \mathrm{~A}^{\circ}$ radius of the co-crystallized reference ligand in the complex crystal structure of the enzyme. Protonation states and tautomer in the binding site are assigned automatically by FlexX and can be adjusted for ambiguous cases. Water molecules can also be managed here.

Assignments already have been calculated by an internal optimization procedure called Protoss. Protoss aims to optimize the hydrogen bond network of the binding site by taking residues, co-factors and the reference ligand as well as selected water molecules, into account but we can make manual adjustments to the hydrogen bond network using the expandable 'Residues', 'Waters' and 'Small Molecule' tables (Lippert and Rarey, 2009).The screened 23 compounds were loaded in FlexX as docking library. Ligand binding driven by enthalpy and entropy hybrid approach is new recommended docking strategy usually generally applicable; combines 'classic triangle matching' with 'single interaction scan' (Jain, 2006). Classic triangle matching tries to place the first fragment of ligand using 3 interactions; often good for hydrophilic ligand and may fail for ligands which cannot form three interactions, e.g., very small fragments and some steroids. Single interaction scan tries to place the first fragment of ligand using 1 interaction only. It takes little longer and is usually best for hydrophobic ligand, small fragment ligands (FBLD), and cases in which classic triangle matching fail (Rarey et al., 1996).

Full score contribution threshold and no score contribution threshold was set as default to 0.30 and 0.70 respectively. The protein ligand clash (maximum allowed overlap volume) and intra ligand clash (clash factor) was set as default to $2.9 \AA$ and $0.6 \AA$ respectively in the docking considering hydrogen in internal clash tests. Stereo mode during docking was considered. Maximum number of solutions per iteration was set to 200 and maximum number of solutions per fragmentation was set to 200. Top 10 poses were kept. The LeadIT suite provides the FlexX-scoring function, which was used to find the initial best poses (Schomburg et al., 2012). For final evaluation of the poses of the ligands affinity toward docked enzyme, the scoring function (Hyde) was used. Hyde is assessment facility of LeadIT software was implemented to report the free energy of binding $(\Delta \mathrm{G})$ and ligand efficiency of the best dock scored pose. Before rescoring, two optimization steps, which are part of the HYDE Module, were applied to each pose (Schneider et al., 2012). In the first step, the software ProToss (Lippert and Rarey, 2009) optimizes the hydrogen bond network of the docking pose. The second step is a geometrical optimization of the pose in the active site minimizing steric clash and conformational strain energy of the ligand while maintaining good hydrogen bond geometries. HYDE is an empirical scoring function, which assesses protein-ligand complex by considering hydrogen bond interactions mainly and also hydrophobic and desolvation effects and provides estimation for the binding affinity (Schneider et al., 2012). HYDE has a low dependence from the size of the docked compound making size correction factors unnecessary (Pan et al., 2003).For scoring analysis; the best FlexX and HYDE score for each compound was taken and compared to the scores of the other compounds and methotrexate. Each compound that has a final score higher than or 
near to the score of methotrexate (also docked and scored) of the enzymatic reaction is estimated as having an inhibiting impact on the enzymatic reaction.

\section{RESULTS AND DISCUSSION}

The first series of target compounds, series A, contain 4(4-substituted phenyl)thiazole-2-amine linked to the 1-position of the triazine ring. 2-Aminothiazole derivatives (1a-c) were synthesized by reacting $\alpha$-bromoacetophenone derivatives with thiourea as shown in Scheme 1(Metwally, 2004).

Alternatively, 2-aminothiazoles derivatives (1d-e) were obtained by condensation reaction between acetophenone derivatives and thiourea in the presence of iodine (Scheme 2) (Rajmane et al., 2013). The second series of target compounds, series B, contain 5-substituted-4-amino-s-trizole-3-thiol linked to triazine ring. Two different strategies were used to prepare the 5alkyl-4-amino-s-trizole-3-thiol and the 5-phenyl-4-amino-s-trizole3-thiol derivatives. Firstly, 5-alkyl-4-amino-s-triazole-3-thiol derivatives were synthesized as shown in Scheme 3. Hydrazine hydrate was reacted with carbon disulfide to afford thiocarbohydrazide (2) in high yield. The corresponding aliphatic monocarboxylic acids were cyclized with thiocarbohydrazide to give the alkytriazole drevatives (3a-d) respectively. Secondly, 5phenyl-4-amino-s-triazole-3-thiol was synthesized as shown in Scheme 4.

The nucleophilic substitution reaction between ethyl benzoate and hydrazine hydrate afforded benzhydrazide (4). The addition reaction between benzhydrazide and carbon disulfide was achieved successfully by using alcoholic potassium hydroxide to give benzoyldithiocarbazate (5). Finally, benzoyldithiocarbazate was cyclized with hydrazine hydrate to afford compound (3e).<smiles>[R]c1ccc(C(=O)CBr)cc1</smiles>

Scheme 1.Synthesis of 2-aminothiazole derivatives (1a-c).

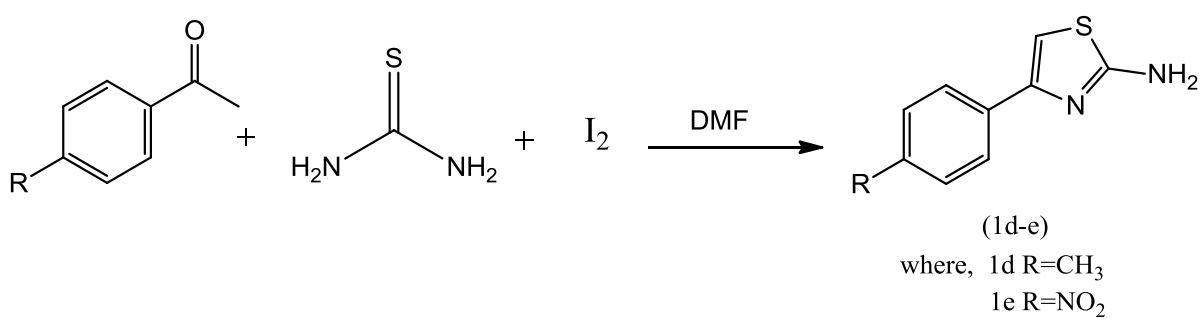

Scheme 2.Synthesis of 2-aminothiazole derivatives (1d-e).

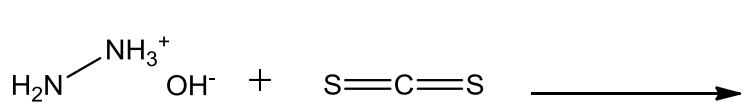<smiles>NNC(=S)NN</smiles>

(2)<smiles>[R]C(=O)O</smiles>

(3a-d)

where, a: $\mathrm{R}=\mathrm{H} ; \mathrm{b}: \mathrm{R}=\mathrm{CH}_{3}$; $\mathrm{c}: \mathrm{R}=\mathrm{C}_{2} \mathrm{H}_{5}$, d: $\mathrm{R}=\mathrm{C}_{4} \mathrm{H}_{9}$ 


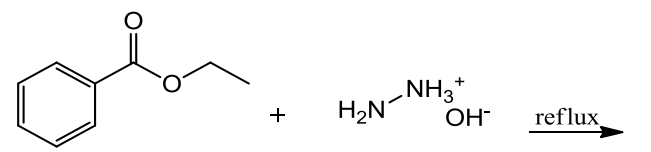<smiles>NNC(=O)c1ccccc1</smiles>

(4)<smiles>Nn1c(S)nnc1-c1ccccc1</smiles>

(3e)

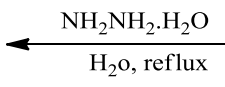

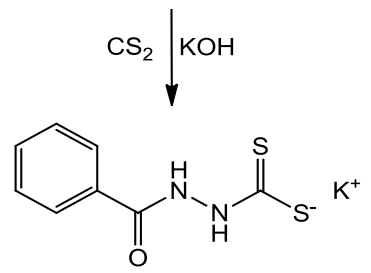

(5)

Scheme 4.Synthesis of 5-phenyl-4-amino-s-triazole-3-thiol (3e).

\section{Mono-substituted triazine derivatives}

The first and the most reactive chlorine atom on cyanuric chloride was substituted with the synthesized heterocyclic amine derivatives (1a-e), (3a-e) or aniline at room temperature to afford the intermediates (6a-e), (7a-e) and (12) respectively as shown in Schemes (5, 6 and 7) respectively. Dichloromethane was the best solvent and $\mathrm{K}_{2} \mathrm{CO}_{3}$ was added to neutralize the evolved $\mathrm{HCl}$ in order to avoid salt formation of the product (Kolmakov, 2008). The synthesized intermediates were characterized by ${ }^{1} \mathrm{HNMR}$ spectroscopy which showed the characteristic peaks for eachproton. In series A (6a-e), 5-CH proton on the thiazole ring appeared between $6.7 \mathrm{ppm}$ and $7.2 \mathrm{ppm}$. In series B (7a-e), characteristic SH proton appeared down field around $12.5 \mathrm{ppm}$. Singlet NH beak appeared between $7.6 \mathrm{ppm}$ and $8.9 \mathrm{ppm}$ in both series.

\section{Synthesis of tri-substituted triazines (target compounds)}

Aiming to synthesize our target compounds of group A, $\mathrm{B}$ and model compounds, nucleophilic substitution of the remaining two chloride atoms was the last step. Different strategies have been tried for the synthesis of our target compounds by controlling the temperature, time, and optimization of variables, such as solvent and base. The correct order of addition of nucleophiles should be followed, taking in consideration the decrease of reactivity with the number of substituents (Afonso et al., 2006). The substitution of the remaining chloride atom with amine required considerably more vigorous conditions (Mathias et al., 1994).

However, the substitution of chloride atom by primary amines was achieved in moderate yield by refluxing the solution in the presence of an excess of the corresponding amine. Other attempts to react with more hindered amines as for example secondary amines under these conditions were unsuccessful (Afonso et al., 2006). Different solvents were used in many reported procedures (Kolmakov, 2008; Solankee et al., 2010) such as 1,4-dioxane, acetone, methylene chloride, tetrahydrofuran, dimethylformamide (DMF) and water. We have tried these solvents in each step of the reaction and methylene chloride was the most suitable for the first step in both series. Solvent with high boiling point was most suitable for heating under reflux for the second step because the substitution of the third chlorine atom in 2,4,6-trichloro-1,3,5-triazine was not an easy task (Mikhaylichenko et al., 2009). In the synthesis of compounds (9a-e and 11a-e), tetrahydrofuran was used as a solvent but in case of compounds (8a-e and 10a-e) water was used. It was not necessary to carry out the reaction of cyanuric chloride with ammonia or amines under anhydrous conditions. The use of an aqueous system did not cause noticeable hydrolysis of the triazine product (Kaiser et al., 1951).
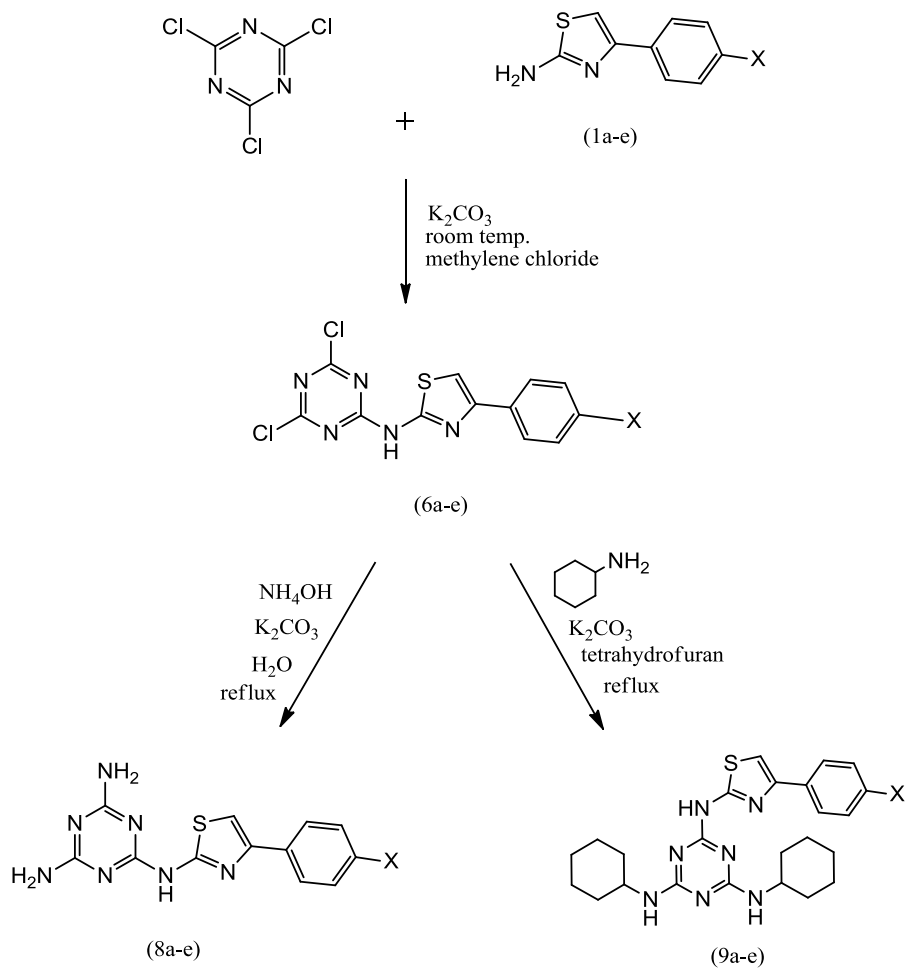

where, a: $\mathrm{X}=\mathrm{H} ; \mathrm{b}: \mathrm{X}=\mathrm{Cl}$; $: \mathrm{X}=\mathrm{OCH}_{3} ; \mathrm{d}: \mathrm{X}=\mathrm{CH}_{3} ; \mathrm{e}: \mathrm{X}=\mathrm{NO}_{2}$

Scheme 5.Synthesis of series A intermediates (6a-e) and target compounds (8a-e, 9a-e).

The reaction in water was incomplete unless cyanuric chloride was used in a finely divided state (Thurston et al., 1951). Excess of the 
amine or adding a base such as sodium hydroxide, sodium carbonate, potassium carbonate or sodium bicarbonate could be used to neutralize the hydrogen chloride formed during each step of the reaction (Kaiser et al., 1951; Thurston et al., 1951).Ammonia and cyclohexylamine were used in excess to replace the two chloride atoms in intermediates (6a-e), (7a-e) and (12) using THF as solvent and heating under reflux under basic condition to afford target compounds (8a-e), (9a-e), (10a-e), (11ae), (13) and (14) respectively as shown in Schemes (5, 6 and 7). This reaction was unsuccessful when equimolar amounts were used in methylene chloride. Isolation of the product by extraction using acidified water: dichloromethane (2:1) was a good strategy to remove excess amine or any salt formed and afforded pure products after evaporation of organic solvent under vacuum. Recrystallization of the solid products from suitable solvent afforded pure compounds.

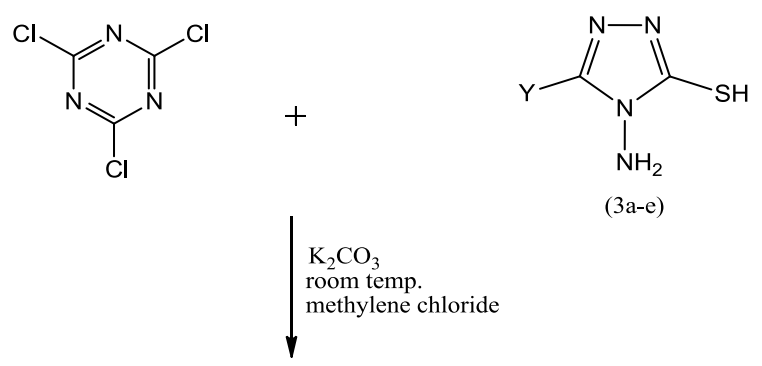<smiles>[Y]c1nnc(S)n1Nc1nc(Cl)nc(Cl)n1</smiles>

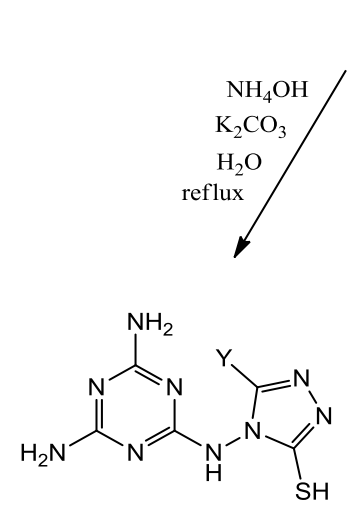

(7a-e) (10a-e)
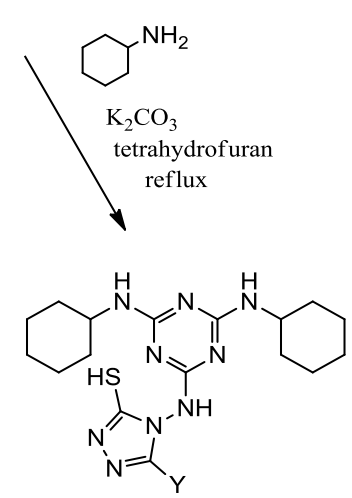

(11a-e) where, a: $\mathrm{Y}=\mathrm{H}$; b: $\mathrm{Y}=\mathrm{CH}_{3}$; : $\mathrm{Y}=\mathrm{C}_{2} \mathrm{H}_{5}$; $\mathrm{Y}: \mathrm{Y}=\mathrm{C}_{4} \mathrm{H}_{9}$; e: $\mathrm{Y}=\mathrm{C}_{6} \mathrm{H}_{5}$

Scheme 6.Synthesis of series B intermediates (7a-e) and targetcompounds (10a-e) and (11a-e).

The synthesized target compounds were characterized by ${ }^{1} \mathrm{H}-\mathrm{NMR}$ and elemental analysis. The synthesized compounds showed characteristic peaks for each proton in ${ }^{1} \mathrm{HNMR}$ spectra. In series A (8a-e) and series B (10a-e) compounds, the spectra were characterized by the singlet peak that represent the four protons of the free amino groups $\left(2 \mathrm{x} \mathrm{NH}_{2}\right)$ which appeared between $2.7 \mathrm{ppm}$ and $3.5 \mathrm{ppm}$. In series A (9a-e) and series B (11a-e) compounds, the spectra were characterized by the protons of cyclohexyl groupthat appeared upfield as a multiplet between $0.9 \mathrm{ppm}$ and 1.7 $\mathrm{ppm}$. The elemental analysis results for the new synthesized compounds were within the accepted limits $( \pm 0.4 \%)$ relative to the calculated values.

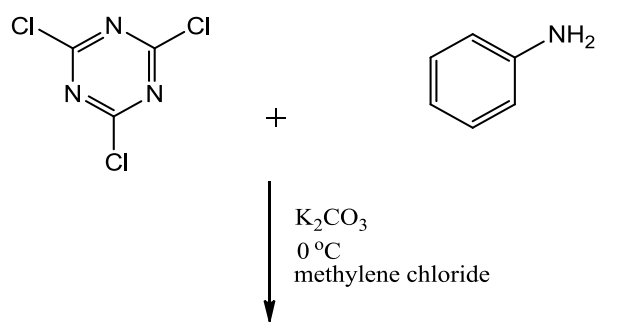<smiles>Clc1nc(Cl)nc(Nc2ccccc2)n1</smiles>

(12)
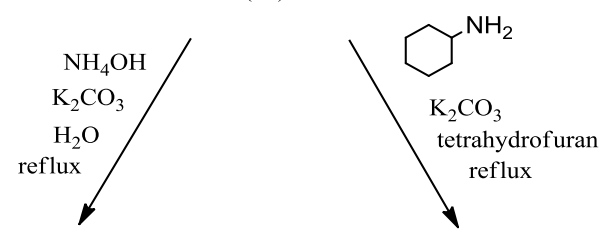<smiles>Nc1nc(N)nc(Nc2ccccc2)n1</smiles>

(13)

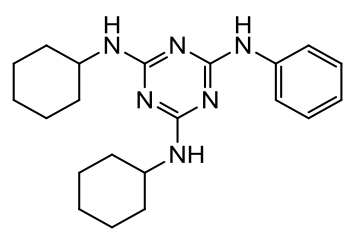

(14)
Scheme 7.Synthesis of model compounds intermediate (12) and target compounds $(\mathbf{1 3}, \mathbf{1 4})$.

\section{Evaluation of cytotoxic activity of target compounds}

In keeping with our scope of investigation, the target novel compounds were evaluated for their cytotoxic activity in vitro using non-small cell lung cancer cell line (A549). Many facts should be considered. First, DHFR enzyme is responsible for synthesis of tetrahydrofolate which is a cofactor necessary for DNA synthesis and repressed expression of DHFR induces cell cycle arrest in human cell lung cancer (A549) (Liado et al., 2009). Second, MTX (X) was reported to be clinically useful DHFR inhibitor (Neradil et al., 2015) and is frequently used in the treatment of cancer (Abolmaali et al., 2013). Finally, the antitumor activity represented as $\mathrm{IC}_{50}$ of MTX $(\mathrm{X})$ was determined in 6 different cancer cell lines and was in an extensively broad range from $6.05 \mathrm{nM}$ to more than $1,000 \mathrm{nM}$. The osteosarcoma (Saos-2) $\left(\mathrm{IC}_{50}>1,000 \mathrm{nM}\right)$ and breast cancer (MCF-7) $\left(\mathrm{IC}_{50}=114.31 \mathrm{nM}\right)$ cells were the most resistant to MTX (X). In contrast, the gastric cancer (AGS) and colon cancer (HCT-116) cells were highly sensitive to MTX (X) with $\mathrm{IC}_{50}$ of $6.05 \mathrm{nM}$ and $13.56 \mathrm{nM}$ respectively. 
Table 1: Structures and in vitro cytotoxic activity of (series A) compounds against (A549) cell line using methotrexate (MTX) as a reference compound.

\begin{tabular}{|c|c|c|}
\hline \multicolumn{3}{|c|}{ where, a:X=H; b: X=Cl; c: X=OCH${ }_{3}$ d: $X=\mathrm{CH}_{3}$ e $: X=\mathrm{NO}_{2}$} \\
\hline Compound No. & Surviving \% & Inhibition \% \\
\hline $8 a$ & 27 & 73 \\
\hline $8 b$ & 38 & 62 \\
\hline $8 \mathrm{c}$ & 30 & 70 \\
\hline $8 \mathrm{~d}$ & 30 & 70 \\
\hline $8 \mathrm{e}$ & 25 & 75 \\
\hline $9 \mathrm{a}$ & 23 & 77 \\
\hline $9 \mathrm{~b}$ & 46 & 54 \\
\hline $9 \mathrm{c}$ & 44 & 56 \\
\hline $9 \mathrm{~d}$ & 50 & 50 \\
\hline $9 \mathrm{e}$ & 29 & 71 \\
\hline MTX & 30 & 70 \\
\hline
\end{tabular}

Table 2: Structures and in vitro cytotoxic activity of (series B) compounds against (A549) cell line.<smiles>[X]c1nnc(S)n1Nc1nc(N)nc(N)n1</smiles>

$10 \mathrm{a}-\mathrm{e}$<smiles>[Y]c1nnc(S)n1Nc1nc(N)nc(N)n1</smiles>

$11 \mathrm{a}-\mathrm{e}$

where, a: $\mathrm{Y}=\mathrm{H} ; \mathrm{b}: \mathrm{Y}=\mathrm{CH}_{3}$; : $\mathrm{Y}=\mathrm{C}_{2} \mathrm{H}_{5} ; \mathrm{d}: \mathrm{Y}=\mathrm{C}_{4} \mathrm{H}_{9} ; \mathrm{e}: \mathrm{Y}=\mathrm{C}_{6} \mathrm{H}_{5}$

\begin{tabular}{ccc}
\hline Compound No. & Surviving $\%$ & Inhibition $\%$ \\
\hline $10 \mathrm{a}$ & 28 & 72 \\
$10 \mathrm{~b}$ & 27 & 73 \\
$10 \mathrm{c}$ & 28 & 72 \\
$10 \mathrm{~d}$ & 27 & 73 \\
$10 \mathrm{e}$ & 25 & 75 \\
$11 \mathrm{a}$ & 33 & 67 \\
$11 \mathrm{~b}$ & 28 & 72 \\
$11 \mathrm{c}$ & 28 & 72 \\
$11 \mathrm{~d}$ & 35 & 65 \\
$1 \mathrm{e}$ & 26 & 74 \\
\hline
\end{tabular}

The two non-small cell lung cancer cell lines, (NCI-H23) and (A549) cells demonstrated similar sensitivity to MTX (X) with $\mathrm{IC}_{50}=38.25 \mathrm{nM}$ and $38.33 \mathrm{nM}$ respectively (Yoon et al., 2010). These findings encouraged us to evaluate in vitro the cytotoxic activity of our target compounds against non-small cell lung carcinoma (A549) cell line using MTX (X) as a reference compound. A549 cell line was widely used as in vitro model for type II pulmonary epithelial cell model for drug metabolism (Sporty et al., 2010). The significance of this search was to develop novel non classical anticancer compounds against lung cancer. The results of single dose experiment $(100 \mu \mathrm{g} / \mathrm{ml})$ of synthesized compounds performed on non-small cell lung carcinoma cell line (A549) are represented in Tables (1-3). Compounds showed percent inhibition less than $70 \%$ are considered inactive as cytotoxic agents (Al-Omar et al., 2005). For (series A) compounds (Table 1), compound (9a) was the most active compound. Four compounds (8a, 8e, 9a and 9e) were more active than MTX while compounds ( $8 \mathrm{c}$ and $8 \mathrm{~d}$ ) were equipotent to MTX. Compounds (8b, 9b, 9c and 9d) were considered inactive against lung cancer cells as they had a lower cytotoxic activity than MTX. In case of group B target compounds (Table 2); compound (10e) was the most active compound.

Eight compounds (10a-e, 11b, 11c and 11e) had a higher cytotoxic activity than MTX against lung cancer cells. Compounds 11a and11d were considered inactive against lung cancer cells as cytotoxic agents. In case of model compounds (Table 3), compound (13) was active while compound (14) was inactive as cytotoxic agent compared to MTX. Interestingly, compound 9a was the best in term of potency against non-small lung cancer cell among the members of this group of inhibitors. As expected, out of the twenty novel synthesized compounds there were twelve compounds showed higher cytotoxic activity than MTX. Two compounds were equipotent to MTX while six compounds were considered inactive. 

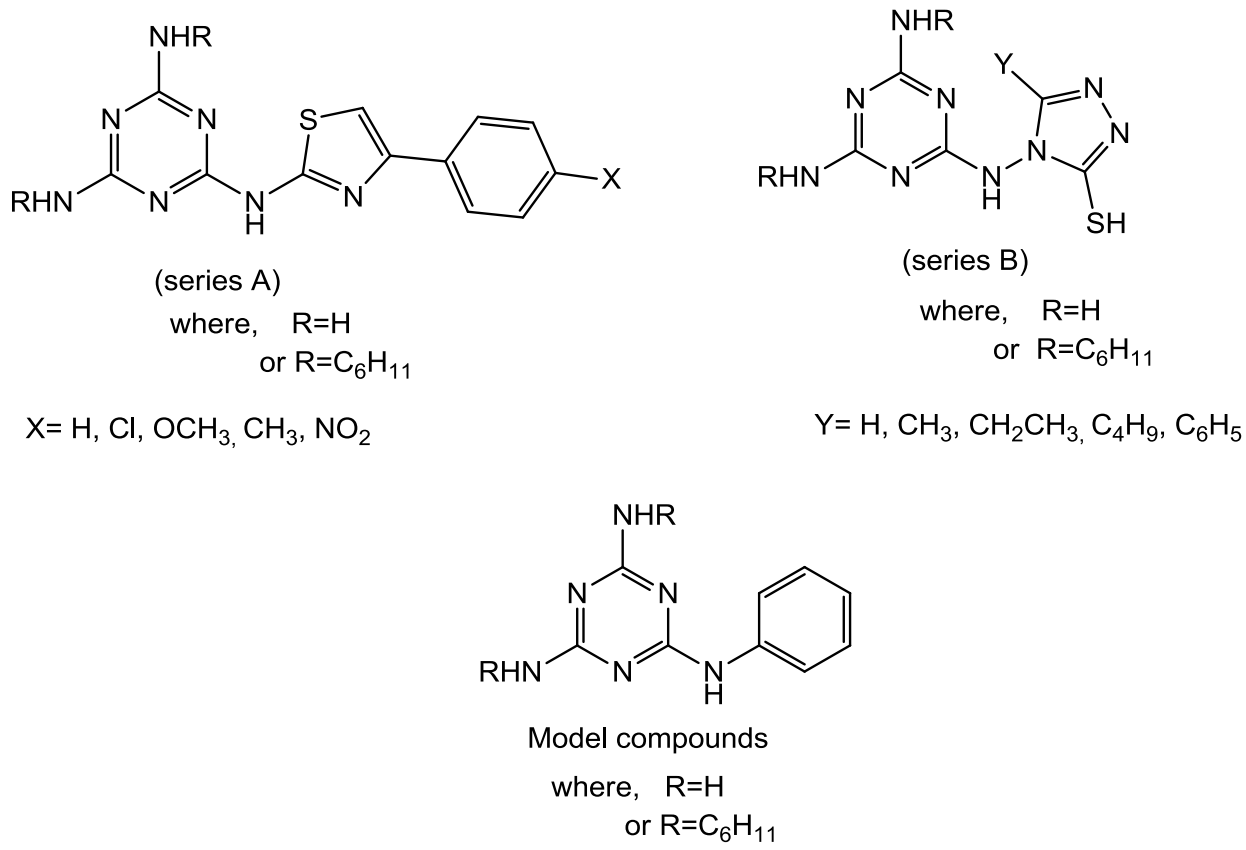

Fig. 1: Chemical structures of target 1,3,5-triazine derivatives (series A \& B) and model compounds.

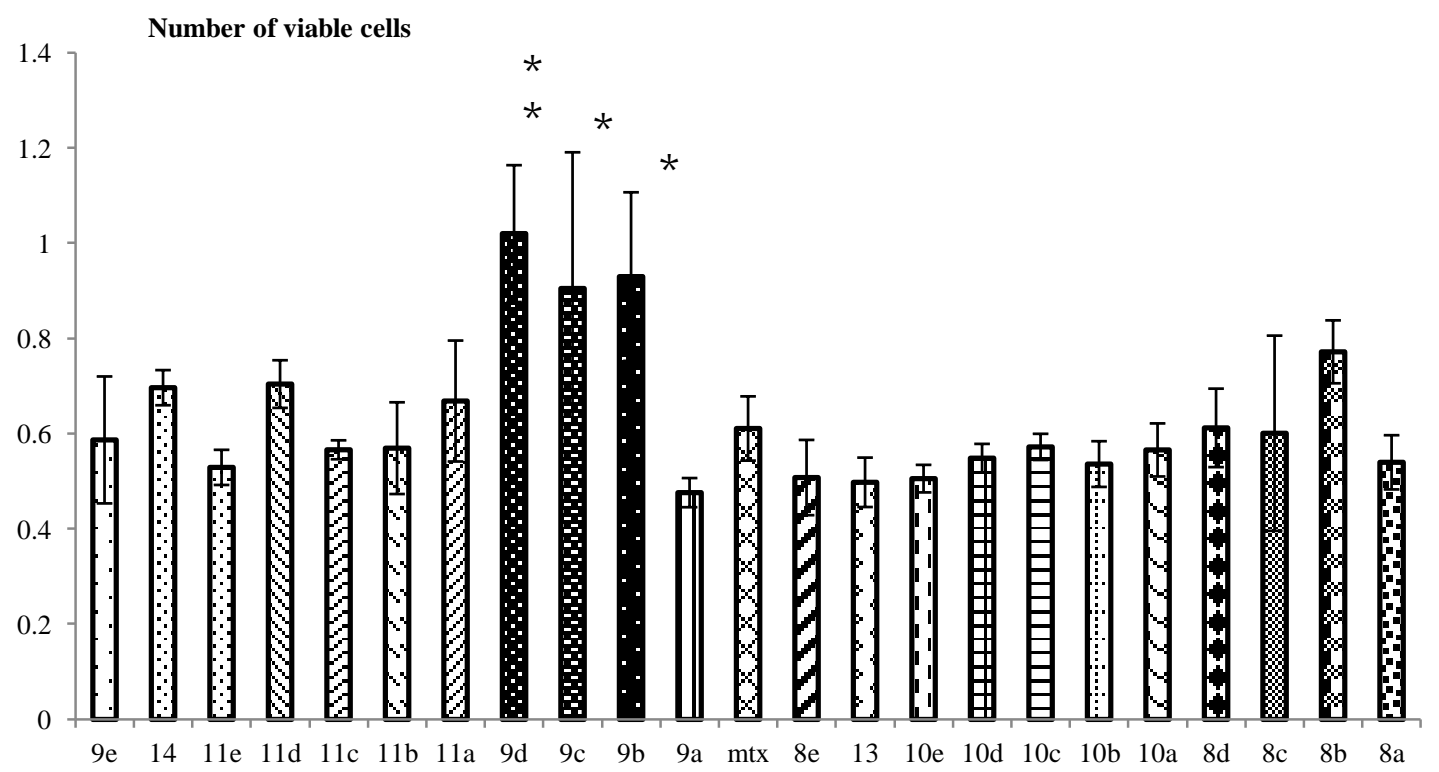

Fig. 2.Statistical analysis of single dose experiment results.

These results reflect that, series $\mathrm{B}$ of synthesized compounds were more active than series A and model compounds. The presence of free amino group on triazine ring enhanced the cytotoxic activity as all compounds in series B having free amino group as well as model compound (13) were more potent as cytotoxic agent than MTX.

Single dose experiment results were statistically analyzed using SPSS 21.0 program (IBM Software, 2015). Differences between compounds were evaluated statistically using one-way
ANOVA (Tukey's test) after normality of distribution had been evaluated by Bartlett's test. The difference was considered significant at $\mathrm{P}<0.05$, and high significant at $\mathrm{P}<0.001$. Statistical analysis (Figure 2) revealed that most of the synthesized compounds showed non-significant difference in their cytotoxic activity relative to MTX except compounds $9 \mathrm{~b}, 9 \mathrm{c}$ and $9 \mathrm{~d}$.The values of $\mathrm{IC}_{50}$ for the most active compounds in each group (8e, 9a, 10e and 11e) (Table 4) were extracted from the graph using Graphpad Prism V6.01 software (GraphPad Software, 2015). 
Table 3: Structures and in vitro cytotoxic activity of model compounds against (A549) cell line.

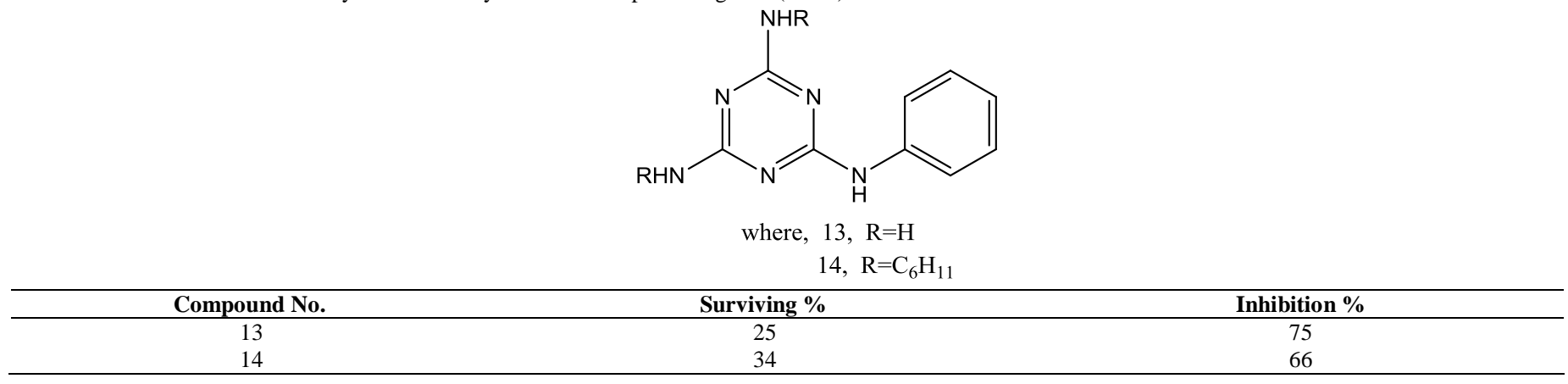

Table 4: Structures and $\mathrm{IC}_{50}$ of the most active target compounds.

\begin{tabular}{|c|c|c|c|}
\hline Compound & $\mathrm{R}$ & $\mathrm{R}^{1}$ & $\mathrm{IC}_{50}(\mathrm{nM})$ \\
\hline $8 \mathrm{e}$ & $\mathrm{H}$ & 4-(4-nitrophenyl)thiazole2-yl & 50 \\
\hline $9 \mathrm{a}$ & $\mathrm{C}_{6} \mathrm{H}_{11}$ & 4-phenylthiazole2-yl & 42 \\
\hline $10 \mathrm{e}$ & $\mathrm{H}$ & 5-phenyl-4H-1,2,4-triazole-3-thiol-4-yl & 62 \\
\hline $11 \mathrm{e}$ & $\mathrm{C}_{6} \mathrm{H}_{11}$ & 5-phenyl-4H-1,2,4-triazole-3-thiol-4-yl & 28 \\
\hline
\end{tabular}

A triplicate experiment was performed for each compound

\section{Evaluation of drug likeliness}

The drug likeliness was evaluated using the Lipinski rule of 5 via Lipinski drug filter protocol (Lipinski, 2004) using Mona software (BioSolveIT GmbH, 2015). Our synthesized target compounds with anticancer activity passed the Lipinski rule of 5 and have properties that would make it a likely orally active drug in humans.

\section{Molecular docking study}

Molecular docking study was performed on synthesized compounds along with the reference molecule, methotrexate, into human DHFR enzyme using FlexX module in LeadIT 2.1.8 software-package (BioSolveIT GmbH, 2014). The 3D structure of hDHFR was studied and was available in the PDB (4m6k) (Protein data bank, 2015). Pose view was the only tool known from the literature that deals with the problem of automatic generation of 2D depictions of complexes. Pose view was operated on a fast tree re-arrangement algorithm to minimize crossing lines in the sketches and was performed well on complexes with ligands which had a molecular weight less than $600 \mathrm{Da}$ (Stierand an Rarey, 2010).

Compounds of series A (8a-e) and series B (10a-e) which contain free amino groups on triazine ring showed interaction pattern in the active site of hDHFR mimic that of MTX as shown in Figures (3-5).In case of series A compounds (8a-e), the two amino group on triazine ring mimic the two amino groups on pteridine ring of MTX in forming hydrogen bonds with a conserved key amino acid residue, Glu 30 (Stockman et al., 1991), and the backbone carbonyl oxygen atoms of Ile 7 and Val 115. Hydrophobic contact of thiazole and phenyl rings with Phe 31, Phe 34, Ser 59 and Ile 60 resembled that of p-aminobenzoic acid moiety of methotrexate (Figures 3,4).

Compounds of series B (10a-e) showed interaction similar to series A (8a-e) and MTX as shown in Figure 5. In case of compound (10e) free amino groups on triazine ring form hydrogen bonds with Glu 30, Ile 7 and Val 115 amino acid residues. Triazole ring and the substituent at 4-position of the triazole ring either aliphatic or aromatic made hydrophobic contact with amino acid residues Phe 31, Phe 34, Thr 56, Val 115, Ile 60, Ala 9 and Leu 67. The larger the substituents on the triazole ring the stronger were the hydrophobic contact with the active site (Figure 5). The strong hydrophobic contact explains the higher cytotoxic activity of compound 10e compared to other compounds in series B (10a-e). Considering series A (9a-e), compound 9e with a nitro group on phenyl ring formed a hydrogen bond with amino acid residues Arg 70 which mimic the interaction of glutamate tail of MTX with hDHFR active site as shown in Figure 6. Compounds containing methyl, methoxy or chloride substitution on phenyl ring showed lower activity because these substitutions were lipophilic in nature and disfavored in this position. Regarding to compounds of series A (9a-e) and series B (11a-e), The two lipophilic cyclohexyl groups showed remarkable hydrophobic contact with Pro 61, Ser 59, and Ile 60 residues as shown in Figure 6 . Interestingly, the presence of two cyclohexyl groups critically enhanced the potency of these analogs by blocking the lipophilic pocket entrance of the active site. 

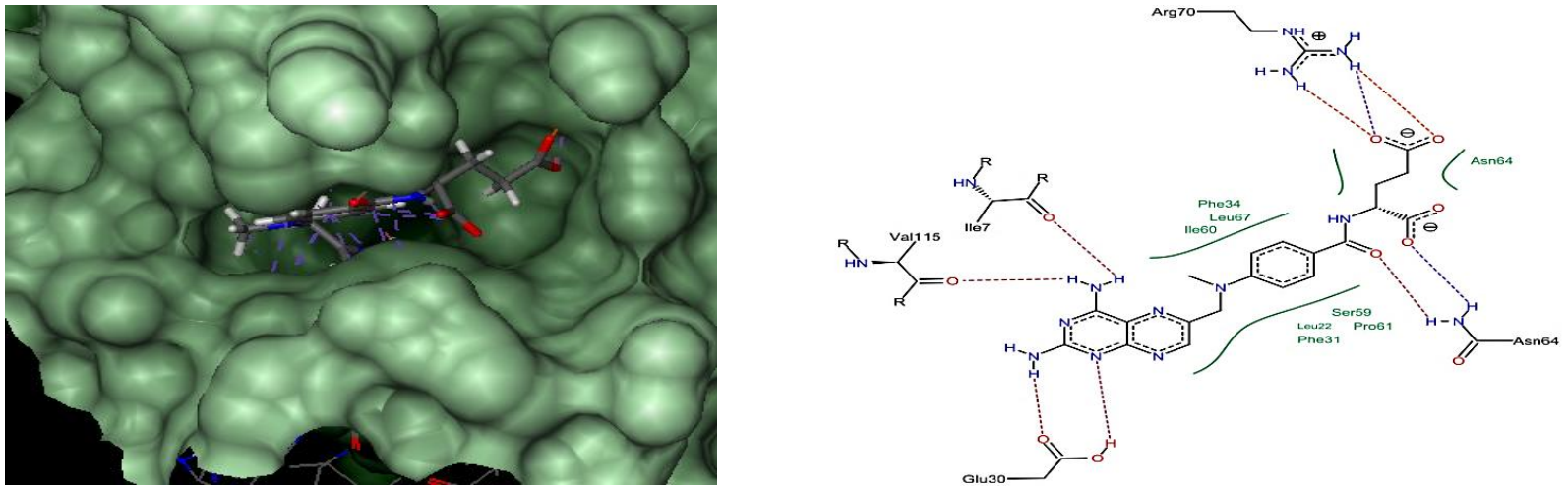

Fig. 3.2D and 3D pose views of MTX docked on the active site of hDHFR
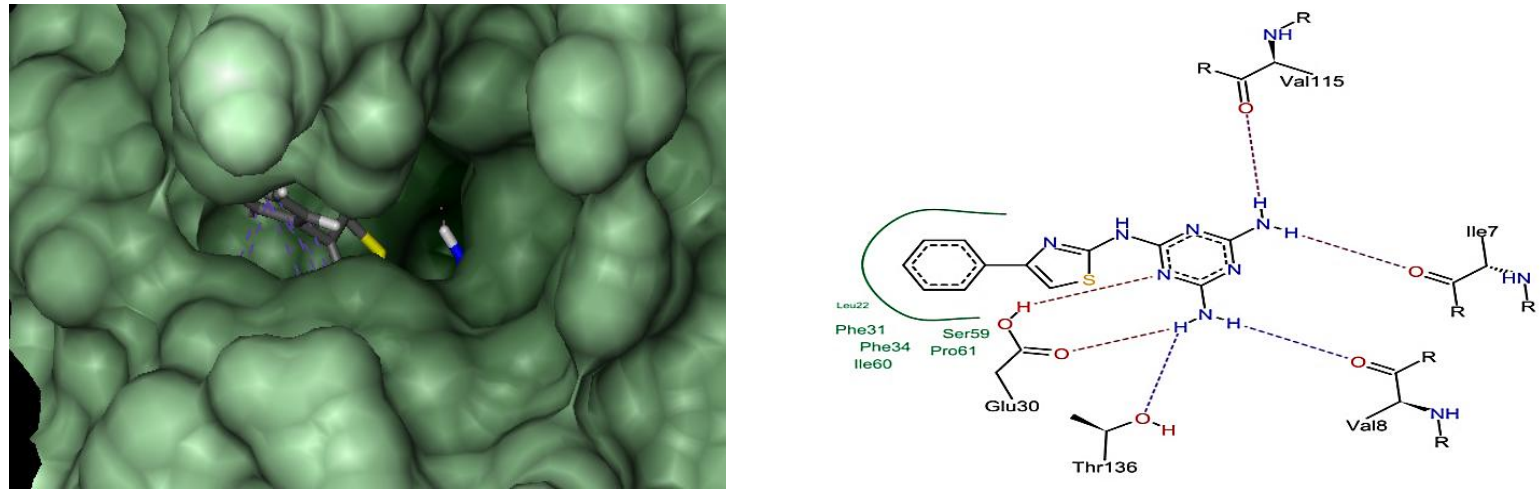

Fig. 4.2D and 3D pose views of compound ( $8 \mathbf{a}$ ) docked on the active site of hDHFR.
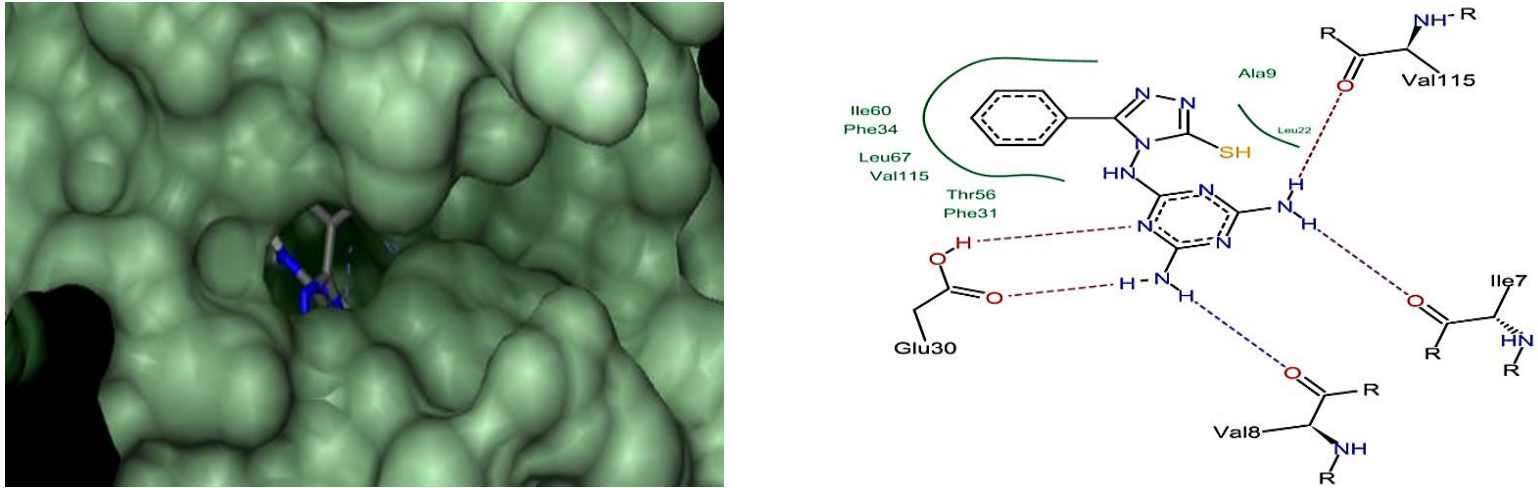

Fig. 5.2D and 3D pose views of compound (10e) docked on the active site of hDHFR.

Careful consideration of compounds in series B (11a-e) revealed that the substituted triazole ring was comparable to the substituted thiazole ring in series A (10a-e) and can access deep in the active site of the enzyme. In series B (11a-e), the activity increased by increasing the number of carbons of alkyl group at 3-position of the triazole ring due to hydrophobic interaction. The absence of or too long alkyl group was associated with decreased activity as observed for compounds $11 \mathrm{a}$ and $11 \mathrm{~d}$.

The optimum binding interaction with active site was observed when the substituent on the triazole ring was the phenyl ring which resulted in the most active compound (11e). It was noticeable that all members of this series showed hydrogen bonding between the two nitrogen atoms of the triazole ring and Asn64 residue like that of glutamate tail of MTX as shown in Figure 7. These results explained the higher activity of series B (11a-e) than series A (9a-e). Compounds (9b-d) did not only lack the hydrogen bonding with Arg70 or Asn64 which resembles glutamate tail of methotrexate but also showed disfavored hydrophobic substituent that making them the least active compounds in all series. Regarding to model compound 14 , it is similar to compound 11a in active site interaction and cytotoxic activity but they differ only in that; in compound $11 \mathrm{a}$, triazole ring make a hydrogen bonding with Asn64 that cannot formed in compound 14, as shown in Figure 8. This difference explained the difference in activity between compound 14 and 11a. 

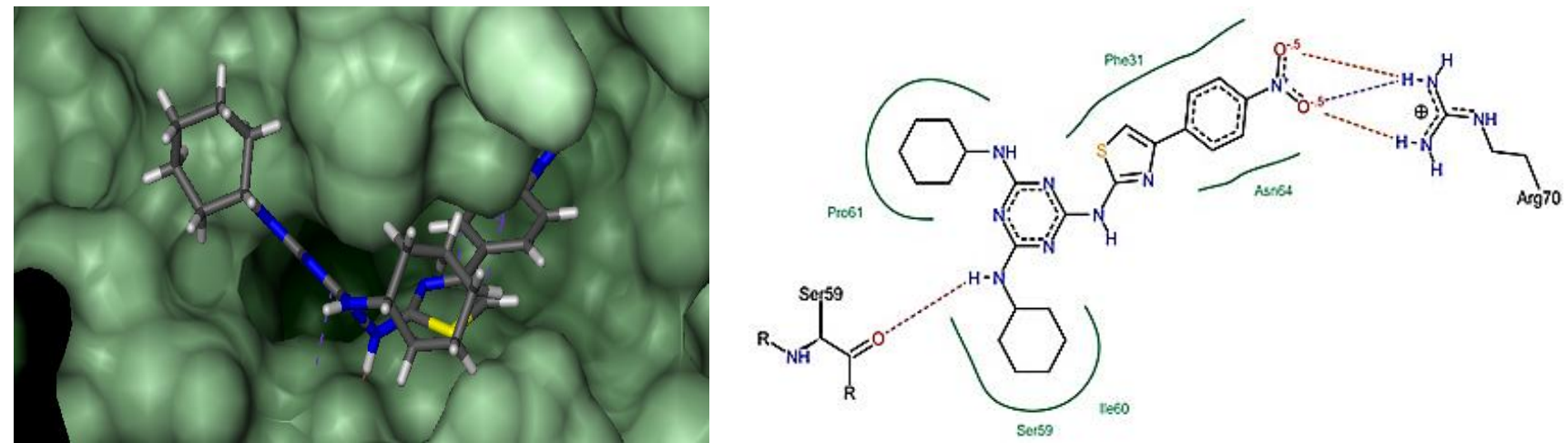

Fig. 6. $2 \mathrm{D}$ and 3D pose views of compound (9e) docked in the active site of hDHFR
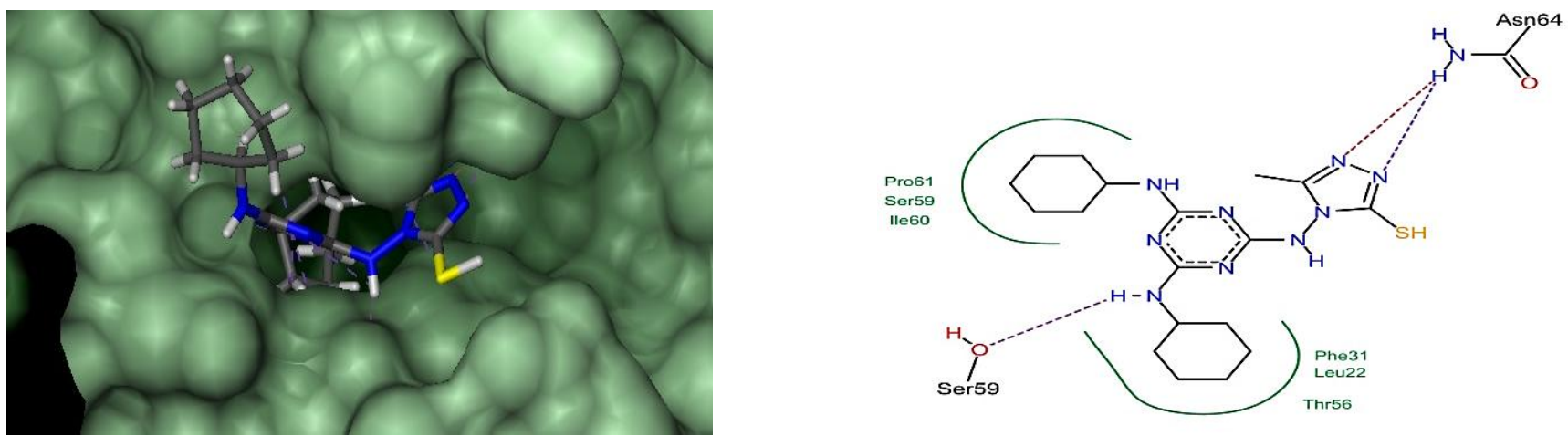

Fig. 7.2D and 3D pose views of compound (11b) docked in the active site of hDHFR
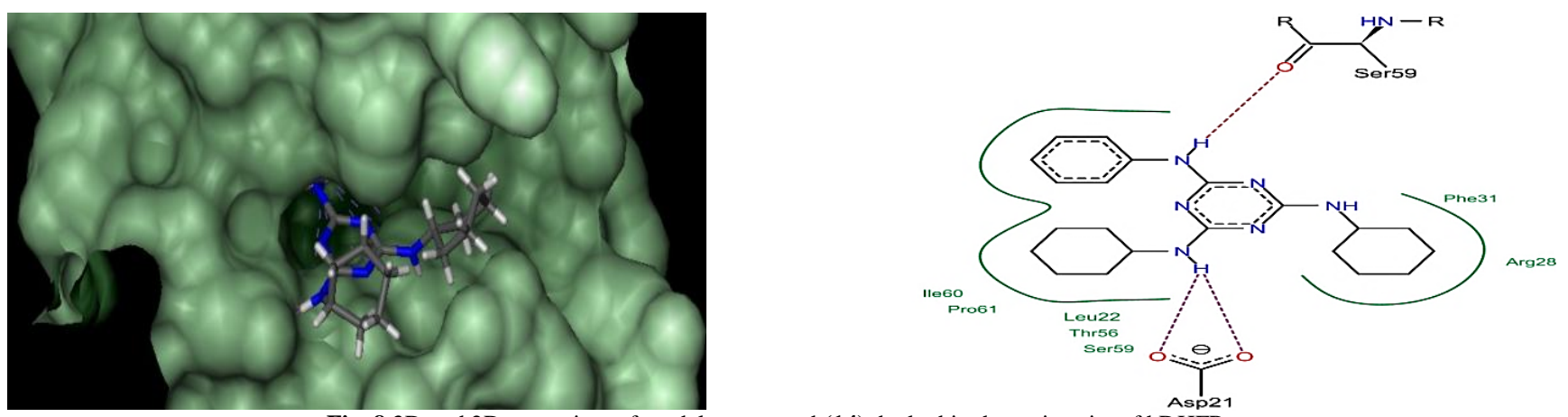

Fig. 8.3D and 2D pose view of model compound (14) docked in the active site of hDHFR.

\section{CONCLUSION}

Two novel series of triazines have been synthesized and evaluated for their cytotoxic activity against non-small cell lung carcinoma cell line (A549).

Our target compounds demonstrated significant anticancer activity against lung cancer compared to methotrexate. Out of twenty two synthesized compounds, there were thirteen compounds showed higher cytotoxic activity than methotrexate.Compounds, $8 \mathrm{e}, 9 \mathrm{a}, 10 \mathrm{e}$ and $11 \mathrm{e}$ were found to be the most potent compounds from both series with a promising cytotoxic activity against lung cancer. Docking study of the synthesized compounds and hDHFR explained the activity differences among the studied compounds taking methotrexate as a reference compound. Docking study demonstrated that some of our target compounds interact with the active site of hDHFR similar to methotrexate.

\section{REFERENCES}

Abolmaali SS, Tamaddon AM, Dinarvand R. A review of therapeutic challenges and achievements of methotrexate delivery systems for treatment of cancer and rheumatoid arthritis. Cancer Chemother Pharmacol, 2013; 71(5): 1115-1130.

Afonso CA, Lourenco NM, Rosatella ADA. Synthesis of 2,4,6tri-substituted-1,3,5-triazines. Molecules, 2006; 11(1): 81-102.

Al-Omar MA, Abdel Hamide SG, Al-Khamees HA, El-Subbagh HI.Synthesis and biological screening of some new substituted-3Hquinazolin-4-one analogs as antimicrobial agents. Saudi Pharmaceut J, 2005; 12: 63-71.

Al-Rashood ST, Hassan GS, El-Messery SM et al. Synthesis, biological evaluation and molecular modeling study of 2-(1,3,4thiadiazolyl-thio and 4-methyl-thiazolyl-thio)-quinazolin-4-ones as a new 
class of DHFR inhibitors. Bioorg. Med Chem Lett, 2014; 24(18): 45574567.

Anderson AC, Wright DL. Antifolate agents: A patent review (2010-2013). Expert opinion on therapeutic patents, 2014; 24(6): 687-697.

Audrieth LF, Scott ES, Kippur PS. Hydrazine derivatives of the carbonic and thiocarbonic acids. I. The preparation and properties of thiocarbohydrazide. J Org Chem, 1954; 19(5): 733-741.

Baker BR, Lourens GJ. Irreversible enzyme inhibitors. Cv.1,2 differential irreversible inhibition of vertebrate dihydrofolic reductases by derivatives of 4,6-diamino-1,2-dihydro-2,2-dimethyl-1-phenyl-s-triazines substituted with a terminal sulfonyl fluoride3. J Med Chem, 1967; 10(6): 1113-1122.

BioSolveIT GmbH "LeadIT.": Available at: http://www.biosolveit.de/FlexX/. [Accessed 4 Augst 2014].

BioSolveIT GmbH. "Mona.": Available at: http://www.biosolveit.de/Mona/. [Accessed 15 April 2015].

Blotny G. Recent applications of 2, 4, 6-trichloro-1, 3, 5-triazine and its derivatives in organic synthesis. Tetrahedron, 2006; 62(41): 95079522.

Böhm HJ. The development of a simple empirical scoring function to estimate the binding constant for a protein-ligand complex of known three-dimensional structure. J Comput Aided Mol Des, 1994; 8(3): 243-256.

Camerman A, Smith HW, Camerman N. Stereochemistry of dihydrofolate reductase inhibitor antitumor acents: Molecular structure of "baker's antifol" (nsc 139105, triazinate) and "insoluble baker's antifol" (nsc 113423). Biochem Biophysical Res Comm, 1978; 83(1): 87-93.

ChemAxon ${ }^{\circledR}$ MarvinSketch: Available at: http://www.chemaxon.com/products/marvin/. [Accessed 15 April 2014].

Daniel B ,Matthias B, Borzilleri RM. 2004. Science of synthesis: Hetarene and related ring systems. Georg Thieme Verlag, Germany .

El-Din NS, El-Fatatry H, Shohyeb M, El-Hmamsy M. Synthesis of some heterocyclic azomethine derivatives of antimicrobial interest. Alexandria Journal of Pharmaceutical Sciences, 1998; 12: 15-20.

El-Hamamsy M, El-Mahdy N. Design, synthesis and biological evaluation of 1,3,5-triazine derivatives as potential inhibitors of dihydrofolate reductase. Life Sci J, 2014; 11(11).

GraphPad Software, Inc. " Graphpad prism V6.01.": Available at: http://www.graphpad.com/scientific-software/prism/. [Accessed 15 April 2015].

Heilbron IM, Bunbury HM. 1934. Dictionary of organic compounds. Eyre and Spottiswoode, London

IBM Software ${ }^{\circledR}(2014)$ "SPSS 21 statistic software": Available at: http://www-01.ibm.com/software/analytics/spss/products/statistics/ features.html. [Accessed 15 April 2015].

Jain AN. Scoring functions for protein-ligand docking. Current Protein and Peptide Science, 2006; 7(5): 407-420.

Kaiser DW, Thurston JT, Dudley JR, Schaefer FC, Hechenbleikner I, Holm-Hansen D. Cyanuric chloride derivatives. II. Substituted melamines. J Am Chem Soc, 1951; 73(7): 2984-2986.

King LC, Hlavacek RJ.The reaction of ketones with iodine and thiourea1. J Am Chem Soc, 1950; 72(8): 3722-3725.

Kolmakov KA. An efficient, "green" approach to aryl amination of cyanuric chloride using acetic acid as solvent. J Het Chem, 2008; 45: 533-539.

Kumar R, Deep Singh A, Singh J, Singh H, Roy R, Chaudhary A. 1,2,3-triazine scaffold as a potent biologically active moiety: A mini review. Mini Rev Med Chem, 2014; 14(1): 72-83.

Llado V, Teres S, Higuera M, Alvarez R, Noguera-Salva M A, Halver J E, Escriba P V, Busquets X. Pivotal role of dihydrofolate reductase knockdown in the anticancer activity of 2-hydroxyoleic acid. Proc Natl Acad Sci U.S.A., 2009;106(33): 13754-13758. $337-341$.

Lipinski C.A., Drug Discovery Today: Technologies, 2004; 1:

Lippert T, Rarey M. Fast automated placement of polar hydrogen atoms in protein-ligand complexes. J cheminform, 2009; 1(1): 112.
Ma X, Chui W-K. Antifolate and antiproliferative activity of 6,8,10-triazaspiro[4.5]deca-6,8-dienes and 1,3,5-triazaspiro[5.5]undeca1,3-dienes. Bioorg Med Chem, 2010; 18(2): 737-743.

Ma X, Tan S-T, Khoo C-L, Sim H-M, Chan L-W, Chui W-K. Synthesis and antimicrobial activity of N1-benzyl or N1-benzyloxy-1,6dihydro-1,3,5-triazine-2,4-diamines. Bioorg Med Chem Lett, 2011; 21(18): 5428-5431.

Manohar S, Khan SI, Rawat DS.4-aminoquinolinetriazine-based hybrids with improved in vitro antimalarial activity against cq-sensitive and cq-resistant strains of plasmodium falciparum. Chem Biol Drug Des, 2013; 81(5): 625-630.

Mathias JP, Seto CT, Simanek EE, Whitesides GM. Selfassembly through hydrogen bonding: Preparation and characterization of three new types of supramolecular aggregates based on parallel cyclic CA3.cntdot.M3 "rosettes". J Am Chem Soc, 1994; 116(5): 1725-1736.

Metwally MA, Abdel-Latif E, Amer FA, Kaupp G. Versatile 2amino-4-substituted-1,3-thiazoles: Synthesis and reactions. J Sul Chem, 2004; 25(1): 63-85.

Mibu N, Yokomizo K, Koga A et al. Synthesis and antiviral activities of some 2, 4, 6-trisubstituted 1,3,5-triazines. Chem Pharm Bull, 2014; 62(10): 1032-1040.

Mikhaylichenko SN, Patel SM, Dalili S, Chesnyuk AA, Zaplishny VN. Synthesis and structure of new 1,3,5-triazine-pyrazole derivatives. Tetrahedron Lett, 2009; 50(21): 2505-2508.

Neradil J, Pavlasova G, Sramek M, Kyr M, Veselska R, Sterba J. DHFR-mediated effects of methotrexate in medulloblastoma and osteosarcoma cells: The same outcome of treatment with different doses in sensitive cell lines. Oncol. Rep, 2015; 33(5): 2169-2175.

Pan Y, Huang N, Cho S, Mackerell AD. Consideration of molecular weight during compound selection in virtual target-based database screening. J Chem Inf Comput Sci, 2003; 43(1): 267-272.

Patel RV, Kumari P, Rajani DP, Pannecouque C, De Clercq E, Chikhalia KH. Antimicrobial, anti-tb, anticancer and anti-hiv evaluation of new s-triazine-based heterocycles. Future med chem, 2012; 4(9): 1053-65.

Protein data bank "PDB Id 4m6k.": Available at: http://www.rcsb.org/pdb/explore/explore.do?structureId=4m6k.

[Accessed 15 March 2015].

Rajmane SV, Lawand AS, Nalawade AM, Bhalvankar RB, Dama LB, G. MP. Microwave assisted synthesis, characterization of substituted 2-aminothiazoles and their biological activity. DAV Int J Sci, 2013; 2(1): 65-69.

Rarey M, Kramer B, Lengauer T, Klebe G. A fast flexible docking method using an incremental construction algorithm. J Mol Biol, 1996; 261(3): 470-489.

Reid JR, Heindel ND. Improved syntheses of 5-substituted-4amino-3-mercapto-(4h)-1,2,4-triazoles. J Het Chem, 1976; 13(4): 925-926.

Saczewski F, Bułakowska A, Bednarski P, Grunert R. Synthesis, structure and anticancer activity of novel 2, 4-diamino-1, 3, 5triazine derivatives. Eur J Med Chem, 2006; 41(2): 219-225.

Sarmah K, Sarmah N, Kurmi K, Patel T. Synthesis and studies of antifungal activity of 2,4,6-trisubstituted 1,3,5-triazines. Adv Appl Sci Res, 2012; 3(3): 1459-1462.

Schneider N, Hindle S, Lange G et al. Substantial improvements in large-scale redocking and screening using the novel HYDE scoring function. J Comput Aided Mol Des, 2012; 26(6): 701-723.

Schomburg KT, Ardao I, Götz K et al. Computational biotechnology: Prediction of competitive substrate inhibition of enzymes by buffer compounds with protein-ligand docking. J Biotechnol, 2012; 161(4): 391-401.

Solankee A, Kapadia K, Ćirić A, Soković M, Doytchinova I, Geronikaki A. Synthesis of some new s-triazine based chalcones and their derivatives as potent antimicrobial agents. Eur J Med Chem, 2010; 45(2): 510-518.

Sporty JL, Horálková L, Ehrhardt C. In vitro cell culture models for the assessment of pulmonary drug disposition. Expert Opin. Drug Metab. Toxicol, 2008; 4(4): 333-345.

Stierand K, Rarey M. Poseview-molecular interaction patterns at a glance. J Cheminform, 2010; 2: 1-1. 
Stockman BJ, Nirmala NR, Wagner G, Delcamp TJ, Deyarman MT, Freisheim JH. Methotrexate binds in a non-productive orientation to human dihydrofolate reductase in solution, based on nmr spectroscopy. FEBS Letters, 1991; 283(2): 267-269.

Sunduru N, Gupta L, Chaturvedi V, Dwivedi R, Sinha S, Chauhan PM. Discovery of new 1, 3, 5-triazine scaffolds with potent activity against mycobacterium tuberculosis h37rv. Eur. J. Med. Chem, 2010; 45(8): 3335-3345.

Thurston JT, Dudley JR, Kaiser DW, Hechenbleikner I, Schaefer FC, Holm-Hansen D. Cyanuric chloride derivatives. I. Aminochloro-s-triazines. J Am Chem Soc, 1951; 73(7): 2981-2983.

Van HTM, Le QM, Lee KY et al. Convenient synthesis of indeno [1,2-c] isoquinolines as constrained forms of 3-arylisoquinolines and docking study of a topoisomerase $\mathrm{i}$ inhibitor into DNA-topoisomerase i complex. Bioorg Med Chem Lett, 2007; 17(21): 5763-5767.

Vichai V, Kirtikara K. Sulforhodamine b colorimetric assay for cytotoxicity screening. Nature protocols, 2006; 1(3): 1112-1116.

World Health Organization "Globocan 2012.": Available at: http://globocan.iarc.fr/Pages/fact_sheets_cancer.aspx. [Accessed 30 March 2015].

World Health Organization "WHO Fact sheet on cancer.": Available at: http://www.who.int/features/factfiles/cancer/en/. [Accessed 30 March 2015].
World Health Organization 'World Cancer Report 2014.": Available at: http://www.iarc.fr/en/publications/books/wcr/. [Accessed 30 March 2015].

Yoon S, Choi JR, Kim J-O, Shin J-Y, Zhang X, Kang J-H. Influence of reduced folate carrier and dihydrofolate reductase genes on methotrexate-induced cytotoxicity. Cancer Treat Res, 2010; 42(3): 163 171.

Zhu W, Liu Y, Zhao Y et al. Synthesis and biological evaluation of novel 6-hydrazinyl-2, 4-bismorpholino pyrimidine and 1,3,5-triazine derivatives as potential antitumor agents. Arch Pharm, 2012; 345(10): 812-821.

\section{How to cite this article:}

Balaha MF, El-Hamamsy MH, El-Din NAS, El-Mahdy NA. Synthesis, Evaluation and Docking Study of 1,3,5-Triazine Derivatives as CytotoxicAgents against Lung Cancer. J App Pharm Sci, 2016; 6 (04): 028-045. 